\title{
Fractional type Marcinkiewicz integral operators associated to surfaces
}

\author{
Yoshihiro Sawano $^{1 *}$ and Kôzô Yabuta²
}

"Correspondence:

yoshihiro-sawano@celery.ocn.ne.jp

${ }^{1}$ Department of Mathematics and

Information Science, Tokyo

Metropolitan University,

Minami-Ohsawa 1-1, Hachioji,

192-0397, Japan

Full list of author information is

available at the end of the article

\begin{abstract}
In this paper, we discuss the boundedness of the fractional type Marcinkiewicz integral operators associated to surfaces, and we extend a result given by Chen et al. (J. Math. Anal. Appl. 276:691-708, 2002). They showed that under certain conditions the fractional type Marcinkiewicz integral operators are bounded from the Triebel-Lizorkin spaces $\dot{F}_{p q}^{\alpha}\left(\mathbb{R}^{n}\right)$ to $L^{p}\left(\mathbb{R}^{n}\right)$. Recently the second author, together with Xue and Yan, greatly weakened their assumptions. In this paper, we extend their results to the case where the operators are associated to the surfaces of the form $\{x=\phi(|y|) y /|y|\} \subset \mathbb{R}^{n} \times\left(\mathbb{R}^{n} \backslash\{0\}\right)$. To prove our result, we discuss a characterization of the homogeneous Triebel-Lizorkin spaces in terms of lacunary sequences.

MSC: Primary 42B20; secondary 42B25; 47G10
\end{abstract}

Keywords: $L^{P}$ boundedness; Marcinkiewicz integral; fractional integral operator; Triebel-Lizorkin spaces; Sobolev spaces

\section{Introduction}

The fractional type Marcinkiewicz operator is defined by

$$
\mu_{\Omega, \rho, \alpha} f(x)=\left(\int_{0}^{\infty}\left|\frac{1}{t^{\rho+\alpha}} \int_{B(t)} f(x-y) \frac{\Omega(y /|y|)}{|y|^{n-\rho}} d y\right|^{2} \frac{d t}{t}\right)^{\frac{1}{2}}
$$

where we write $B(r)=\{|x|<r\} \subset \mathbb{R}^{n}$ for $r>0$ here and below. The operator $\mu_{\Omega, \rho, \alpha} f$ is the so called singular integral operator. In this paper, we shall prove that this operator is bounded under a certain highly weak integrability assumption. To this end, we plan to employ a modified Littlewood-Paley decomposition adapted to our situation. It turns out that we can relax the integrability assumption on $\Omega$ and that the integral operator itself can be generalized to a large extent.

Let $S^{n-1}$ be the unit sphere in the $n$-dimensional Euclidean space $\mathbb{R}^{n}(n \geq 2)$, with the induced Lebesgue measure $d \sigma=d \sigma\left(x^{\prime}\right)$ and $\Omega \in L^{1}\left(S^{n-1}\right)$. In the sequel, we often suppose that $\Omega$ satisfies the cancellation condition

$$
\int_{S^{n-1}} \Omega\left(y^{\prime}\right) d \sigma\left(y^{\prime}\right)=0 .
$$

Here, for the symbols $x^{\prime}$ and $y^{\prime}$, we adopt the following convention: Sometimes they stand for points in $S^{n-1}$. But for $x \in \mathbb{R}^{n} \backslash\{0\}$, we abbreviate $x /|x|$ to $x^{\prime}$ in the present paper. We make this slight abuse of notation since no confusion is likely to occur.

○2014 Sawano and Yabuta; licensee Springer. This is an Open Access article distributed under the terms of the Creative Commons Attribution License (http://creativecommons.org/licenses/by/2.0), which permits unrestricted use, distribution, and reproduction in any medium, provided the original work is properly cited. 
In the present paper we deal with operators of Marcinkiewicz type. Define

$$
\mu_{\Omega, \rho, \alpha, q} f(x):=\left(\int_{0}^{\infty}\left|\frac{1}{t^{\rho+\alpha}} \int_{B(t)} f(x-y) \frac{\Omega\left(y^{\prime}\right)}{|y|^{n-\rho}} d y\right|^{q} \frac{d t}{t}\right)^{\frac{1}{q}} \quad\left(x \in \mathbb{R}^{n}\right) .
$$

As a special case, by letting $\rho=1, \alpha=0, q=2$, we recapture the Marcinkiewicz integral operator that Stein introduced in 1958 [1]. In 1960, Hörmander considered the parametric Marcinkiewicz integral operator $\mu_{\Omega, \rho, \alpha, 2}$ [2]. Since then, about Marcinkiewicz type integral operators, many works appeared. A nice survey is given by Lu [3].

We formulate our results in the framework of Triebel-Lizorkin spaces of homogeneous type. For $\alpha \in \mathbb{R}$ and $p, q \in(1, \infty)$, we let $\dot{F}_{p q}^{\alpha}\left(\mathbb{R}^{n}\right)$ be the Triebel-Lizorkin space defined in $[4,5]$. Note that the space $\mathcal{S}_{\infty}\left(\mathbb{R}^{n}\right)$ given by

$$
\mathcal{S}_{\infty}\left(\mathbb{R}^{n}\right):=\bigcap_{\alpha \in(\mathbb{N} \cup\{0\})^{n}}\left\{f \in \mathcal{S}\left(\mathbb{R}^{n}\right): \int_{\mathbb{R}^{n}} x^{\alpha} f(x) d x=0\right\}
$$

is dense in $\dot{F}_{p q}^{\alpha}\left(\mathbb{R}^{n}\right)$ as long as $\alpha \in \mathbb{R}$ and $p, q \in(1, \infty)$. If $u \in(1, \infty)$, then define $u^{\prime}=\frac{u}{u-1}$ and $\tilde{u}=\max \left(u, u^{\prime}\right)$. Here and below a tacit understanding in the present paper is that the letter $C$ is used for constants that may change from one occurrence to another, that is, the letter $C$ will denote a positive constant which may vary from line to line but will remain independent of the relevant quantities. Our main theorem in the simplest form reads as follows:

Theorem 1 Let $\rho>0,1<p, q<\infty$ and $\Omega \in L^{1}\left(S^{n-1}\right)$.

(i) If $\alpha \in(0,4 /(\tilde{p} \tilde{q}))$ and $\Omega$ satisfies the cancellation condition (1.2), then

$$
\left\|\mu_{\Omega, \rho, \alpha, q} f\right\|_{L^{p}\left(\mathbb{R}^{n}\right)} \leq C\|\Omega\|_{L^{1}\left(S^{n-1}\right)}\|f\|_{\dot{F}_{p q}^{\alpha}\left(\mathbb{R}^{n}\right)}
$$

for all $f \in \mathcal{S}_{\infty}\left(\mathbb{R}^{n}\right)$.

(ii) If $\alpha \in\left(-\min \left\{\frac{4 \beta}{\tilde{p} \tilde{q}}, \rho\right\}, 0\right)$ and

$$
Z_{\Omega}:=\sup _{\xi^{\prime} \in S^{n-1}} \int_{S^{n-1}} \frac{\left|\Omega\left(y^{\prime}\right)\right|}{\left|y^{\prime} \cdot \xi^{\prime}\right|^{\beta}} d \sigma\left(y^{\prime}\right)<+\infty
$$

for some $0<\beta \leq 1$, then

$$
\left\|\mu_{\Omega, \rho, \alpha, q} f\right\|_{L^{p}\left(\mathbb{R}^{n}\right)} \leq C Z_{\Omega}\|f\|_{\dot{F}_{p q}^{\alpha}\left(\mathbb{R}^{n}\right)}
$$

for all $f \in \mathcal{S}_{\infty}\left(\mathbb{R}^{n}\right)$.

(iii) If $\alpha=0$ and $\Omega \in L \log L\left(S^{n-1}\right)$ satisfies the cancellation condition (1.2), then

$$
\left\|\mu_{\Omega, \rho, \alpha, q} f\right\|_{L^{p}\left(\mathbb{R}^{n}\right)} \leq C\|\Omega\|_{L \log L\left(S^{n-1}\right)}\|f\|_{\dot{F}_{p q}^{\alpha}\left(\mathbb{R}^{n}\right)}
$$

for all $f \in \mathcal{S}_{\infty}\left(\mathbb{R}^{n}\right)$.

In any case, by density we can extend (1.4), (1.6) and (1.7) and have them for all $\in \dot{F}_{p q}^{\alpha}\left(\mathbb{R}^{n}\right)$.

In 2002, Chen et al. obtained a result about the fractional type Marcinkiewicz integral operator [6], which we recall now. 
Theorem A Let $1<p, q<\infty$ and $1<r \leq \infty$. Suppose $\Omega \in L^{r}\left(S^{n-1}\right)$ satisfies the cancellation condition (1.2). If $|\alpha|<2 /\left(r^{\prime} \tilde{p} \tilde{q}\right)$ and $\rho=1$, then

$$
\left\|\mu_{\Omega, \rho, \alpha, q} f\right\|_{L^{p}\left(\mathbb{R}^{n}\right)} \leq C\|\Omega\|_{L^{r}\left(S^{n-1}\right)}\|f\|_{\dot{F}_{p q}^{\alpha}\left(\mathbb{R}^{n}\right)}
$$

for all $f \in \mathcal{S}_{\infty}\left(\mathbb{R}^{n}\right)$.

$\mathrm{Si}$, Wang and Jiang discussed ones of somewhat different type [7]. About Theorems 1 and $\mathrm{A}$, a couple of remarks may be in order.

Remark 1 If $0<\beta<1,1 /(1-\beta)<r \leq \infty$ and $\Omega \in L^{r}\left(S^{n-1}\right)$, it is easily seen that the condition (1.5) is satisfied. In this case

$$
Z_{\Omega} \leq C\|\Omega\|_{L^{r}\left(S^{n-1}\right)} .
$$

So, our result includes completely Theorem A, where they assumed that $\Omega \in L^{r}\left(S^{n-1}\right)$. Let $r>1$ and define

$$
\Omega_{0}\left(y^{\prime}\right)=\operatorname{sgn}\left(y^{\prime} \cdot(1,0, \ldots, 0)\right)\left|\left(y^{\prime} \cdot(1,0, \ldots, 0)\right)\right|^{-1 / r} .
$$

Then it is also easily checked that $\Omega$ is in $L^{1}\left(S^{n-1}\right) \backslash L^{r}\left(S^{n-1}\right)$ and satisfies (1.5) for any $\beta \in$ $\left(0,1 / r^{\prime}\right)$.

In the case $\alpha=0, \rho=1$ and $q=2$, the conclusion in Theorem 1(iii) is shown to hold even when $\Omega \in L \log L^{1 / 2}\left(S^{n-1}\right)$ in [8].

Remark 2 We can relax the condition on $\alpha:|\alpha|<4 /\left(r^{\prime} \tilde{p} \tilde{q}\right)$ suffices. Indeed, one can get $\left|\left(\Omega(\cdot)|\cdot|^{-n+1} \chi_{B(1)}\right)(\xi)\right| \leq C|\xi|^{-1 / r^{\prime}}$ by direct computation.

By reexamining their proof, we can parametrize Theorem A: we can prove

$$
\left(\int_{\mathbb{R}^{n}}\left(\int_{0}^{\infty}\left|\frac{1}{t^{\rho+\alpha}} \int_{B(t)} f(x-y) \frac{\Omega(y)}{|y|^{n-\rho}} d y\right|^{q} \frac{d t}{t}\right)^{p / q} d x\right)^{1 / p} \leq C\|f\|_{\dot{F}_{p q}^{\alpha}\left(\mathbb{R}^{n}\right)}
$$

provided $|\alpha|<4 \min \left\{\frac{1}{r^{\prime}}, \min (\rho, 1)\right\} \frac{1}{\tilde{\tilde{q} \tilde{q}}}$. Comparing (1.9) with Theorem 1 , one concludes that our theorem outranges Theorem $\mathrm{A}$ in view of the case when $\min (\rho, 1)<1 / r^{\prime}$. In our earlier paper [9], we improved Theorem A by relaxing the conditions postulated on $\Omega$.

Our method is also applicable even in more generalized settings. For $\rho>0, \alpha \in \mathbb{R}$ and $\Omega \in L^{1}\left(S^{n-1}\right)$, we define the fractional type Marcinkiewicz integral operator by (1.1) and the fractional type Marcinkiewicz integral operator associated to surfaces $\{(x, y): x=$ $\left.\phi(|y|) y^{\prime}\right\} \in \mathbb{R}^{n} \times\left(\mathbb{R}^{n} \backslash\{0\}\right)$ by

$$
\mu_{\Omega, \rho, \phi, \alpha} f(x)=\left(\int_{0}^{\infty}\left|\frac{1}{t^{\rho} \phi(t)^{\alpha}} \int_{B(t)} f\left(x-\phi(|y|) y^{\prime}\right) \frac{\Omega(y)}{|y|^{n-\rho}} d y\right|^{2} \frac{d t}{t}\right)^{\frac{1}{2}} .
$$

Theorem 1 extends further to the case when the operator is equipped with a function space $\Delta_{\gamma}$ with $\gamma \geq 1$. Regarding to Calderón-Zygmund singular integral and Marcin- 
kiewicz integral operators, many authors discussed those operators with modified kernel $b(|\cdot|) \Omega(\cdot)$ in place of $\Omega(\cdot)$, where $b$ belongs to the class of all measurable functions $h:[0, \infty) \rightarrow \mathbb{C}$ satisfying $\|h\|_{\Delta_{\gamma}}=\sup _{R>0}\left(R^{-1} \int_{0}^{R}|h(t)|^{\gamma} d t\right)^{1 / \gamma}<\infty(1 \leq \gamma \leq \infty)$, see [1014], etc. We note that

$$
L^{\infty}\left(\mathbb{R}_{+}\right) \subset \Delta_{\beta}\left(\mathbb{R}_{+}\right) \subset \Delta_{\alpha}\left(\mathbb{R}_{+}\right) \quad \text { for } 1 \leq \alpha<\beta,
$$

and that all these inclusions are proper. We refer to [15-17] for extension and generalization of the space $\Delta_{\gamma}$.

We define the modified fractional type Marcinkiewicz operator $\mu_{\Omega, \rho, \alpha, q}^{(b)}$ by

$$
\mu_{\Omega, \rho, \alpha, q}^{(b)} f(x)=\left(\int_{0}^{\infty}\left|\frac{1}{t^{\rho+\alpha}} \int_{B(t)} f(x-y) \frac{b(|y|) \Omega\left(y^{\prime}\right)}{|y|^{n-\rho}} d y\right|^{q} \frac{d t}{t}\right)^{\frac{1}{q}} .
$$

We can recover Theorem 1 by letting $b \equiv 1$ in the next theorem.

Theorem 2 Suppose that we are given $\Omega \in L^{1}\left(S^{n-1}\right)$ and parameters $p, q, \alpha, \gamma, \rho$ satisfying

$$
1<p, q<\infty, \quad \gamma>\frac{1}{2} \max \{\tilde{p}, \tilde{q}\}, \quad \rho>0 .
$$

(i) Let $\alpha \in\left(0, \frac{4(1 / \tilde{p}-1 /(2 \gamma))(1 / \tilde{q}-1 /(2 \gamma))}{(1-1 / \gamma)^{2}}\right)$. If $b \in \Delta_{\gamma}\left(\mathbb{R}_{+}\right)$and $\Omega$ satisfies the cancellation condition (1.2), then

$$
\left\|\mu_{\Omega, \rho, \alpha, q}^{(b)} f\right\|_{L^{p}\left(\mathbb{R}^{n}\right)} \leq C\|\Omega\|_{L^{1}\left(S^{n-1}\right)}\|b\|_{\Delta_{\gamma}}\|f\|_{\dot{F}_{p q}^{\alpha}\left(\mathbb{R}^{n}\right)}
$$

for all $f \in \mathcal{S}_{\infty}\left(\mathbb{R}^{n}\right)$.

(ii) Assume $\alpha \in\left(-\min \left\{2 \beta \frac{1 / \tilde{p}-1 /(2 \gamma)}{1-1 / \gamma} \cdot \frac{1 / \tilde{q}-1 /(2 \gamma)}{1-1 / \gamma}, \rho\right\}, 0\right)$ with $\beta \in(0,1]$. If $b \in \Delta_{\max (\gamma, 2)}$ and

$$
W_{\Omega}:=\sqrt{\sup _{\xi^{\prime} \in S^{n-1}} \int_{S^{n-1} \times S^{n-1}} \frac{\left|\Omega\left(y^{\prime}\right) \Omega\left(z^{\prime}\right)\right|}{\left|\left(y^{\prime}-z^{\prime}\right) \cdot \xi^{\prime}\right|^{\beta}} d \sigma\left(y^{\prime}\right) d \sigma\left(z^{\prime}\right)}<+\infty,
$$

then

$$
\left\|\mu_{\Omega, \rho, \alpha, q}^{(b)} f\right\|_{L^{p}\left(\mathbb{R}^{n}\right)} \leq C W_{\Omega}\|b\|_{\max (\gamma, 2)}\|f\|_{\dot{F}_{p q}^{\alpha}\left(\mathbb{R}^{n}\right)}
$$

for all $f \in \mathcal{S}_{\infty}\left(\mathbb{R}^{n}\right)$.

(iii) Assume $\alpha=0$. If $b \in \Delta_{\max (\gamma, 2)}, \Omega \in L \log L\left(S^{n-1}\right)$ and $\Omega$ satisfies the cancellation condition (1.2), then

$$
\left\|\mu_{\Omega, \rho, \alpha, q}^{(b)} f\right\|_{L^{p}\left(\mathbb{R}^{n}\right)} \leq C\|\Omega\|_{L \log L\left(S^{n-1}\right)}\|b\|_{\max (\gamma, 2)}\|f\|_{\dot{F}_{p q}^{\alpha}\left(\mathbb{R}^{n}\right)}
$$

for all $f \in \mathcal{S}_{\infty}\left(\mathbb{R}^{n}\right)$. 
In any case, by density we can extend (1.12), (1.14) and (1.15) and have them for all $f \in$ $\dot{F}_{p q}^{\alpha}\left(\mathbb{R}^{n}\right)$.

Remark 3 In Theorem 1(ii) a modification of the proof changes $4 \beta$ into $2 \beta$. We cannot estimate directly the Fourier transform of the measure $\sigma_{t}$ in Section 3, and we use the idea given by Duoandikoetxea and Rubio de Francia [11, p.551] as in Chen et al. [6].

If $0<\beta<1,1 /(1-\beta)<r \leq \infty$ and $\Omega \in L^{r}\left(S^{n-1}\right)$, it is easily seen that the condition (1.13) is satisfied. In this case

$$
W_{\Omega} \leq C\|\Omega\|_{L^{r}\left(S^{n-1}\right)} .
$$

In the case $\alpha=0, \rho=1$ and $q=2$, it is again known in [18] that the conclusion in Theorem 2(iii) holds even when $\Omega \in L \log L^{1 / 2}\left(S^{n-1}\right)$.

In the earlier paper [9], in Theorem 1(ii) (respectively, in Theorem 2(ii)), we needed to postulate the additional conditions $\rho>\beta$ (respectively, $2 \rho>\beta$ ) and the cancellation condition on $\Omega$. However, these are no longer necessary in the new theorems.

Remark In [19], instead of $W_{\Omega}$, the following quantity is proposed:

$$
\sup _{\xi^{\prime} \in S^{n-1}}\left|\int_{S^{n-1}} \int_{S^{n-1}} \Omega\left(y^{\prime}\right) \overline{\Omega\left(z^{\prime}\right)} \log \left(\frac{2}{\left|\xi^{\prime} \cdot y^{\prime}\right|^{2}+\left|\xi^{\prime} \cdot z^{\prime}\right|^{2}}\right)^{1 / 2} d \sigma\left(y^{\prime}\right) d \sigma\left(z^{\prime}\right)\right|<\infty .
$$

In addition to the factor of $b$, we can even distort the convolution. For $\alpha>0,1 \leq q<\infty$, a kernel $\Omega$ and a positive function $\phi$ on $\mathbb{R}_{+}$, we define the operator $\mu_{\Omega, \rho, \phi, \alpha, q}$ and the modified one $\mu_{\Omega, \rho, \phi, \alpha, q}^{(b)}$ by

$$
\mu_{\Omega, \rho, \phi, \alpha, q} f(x)=\left(\int_{0}^{\infty}\left|\frac{1}{t^{\rho} \phi(t)^{\alpha}} \int_{B(t)} f\left(x-\phi(|y|) y^{\prime}\right) \frac{\Omega(y)}{|y|^{n-\rho}} d y\right|^{q} \frac{d t}{t}\right)^{\frac{1}{q}},
$$

and

$$
\mu_{\Omega, \rho, \phi, \alpha, q}^{(b)} f(x)=\left(\int_{0}^{\infty}\left|\frac{1}{t^{\rho} \phi(t)^{\alpha}} \int_{B(t)} f\left(x-\phi(|y|) y^{\prime}\right) \frac{b(|y|) \Omega(y)}{|y|^{n-\rho}} d y\right|^{q} \frac{d t}{t}\right)^{\frac{1}{q}} .
$$

Now we formulate our main theorem. Here and below we write $\mathbb{R}_{+}:=(0, \infty)$.

Theorem 3 Let $\rho>0,1<p, q<\infty$ and $\Omega \in L^{1}\left(S^{n-1}\right)$. Let $c_{0}>1$ and $c_{1}>0$. Suppose that $\phi: \mathbb{R}_{+} \rightarrow \mathbb{R}_{+}$is a nonnegative increasing $C^{1}$-function such that

$$
\phi(2 t) \leq c_{0} \phi(t) \quad \text { for all } t \in \mathbb{R}_{+}
$$

and that

$$
\phi(t) \leq c_{1} t \phi^{\prime}(t) \quad \text { for all } t \in \mathbb{R}_{+} .
$$

Define

$$
\varphi(t):=\frac{\phi(t)}{t \phi^{\prime}(t)} \quad \text { for all } t \in \mathbb{R}_{+} .
$$


Then:

(i) Let

$$
\alpha \in\left(0, \frac{4}{\tilde{p} \tilde{q} c_{1} \log _{2} c_{0}}\right)
$$

If $\Omega$ satisfies the cancellation condition (1.2), then

$$
\left\|\mu_{\Omega, \rho, \phi, \alpha, q} f\right\|_{L^{p}\left(\mathbb{R}^{n}\right)} \leq C\|\Omega\|_{L^{1}\left(S^{n-1}\right)}\|f\|_{\dot{F}_{p q}^{\alpha}\left(\mathbb{R}^{n}\right)}
$$

for all $f \in \mathcal{S}_{\infty}\left(\mathbb{R}^{n}\right)$.

(ii) Let

$$
\alpha \in\left(-\min \left\{\frac{4 \beta}{c_{1} \log _{2} c_{0} \cdot \tilde{p} \tilde{q}}, \frac{\rho}{\log _{2} c_{0}}\right\}, 0\right) .
$$

If $\phi$ satisfies the following additional condition:

$$
\varphi(t) \text { or } t \phi^{\prime}(t) \text { is monotonic on } \mathbb{R}_{+} \text {, }
$$

and $\Omega$ satisfies

$$
Z_{\Omega}:=\sup _{\xi^{\prime} \in S^{n-1}} \int_{S^{n-1}} \frac{\left|\Omega\left(y^{\prime}\right)\right|}{\left|y^{\prime} \cdot \xi^{\prime}\right|^{\beta}} d \sigma\left(y^{\prime}\right)<+\infty,
$$

for some $0<\beta \leq 1$, then

$$
\left\|\mu_{\Omega, \rho, \phi, \alpha, q} f\right\|_{L^{p}\left(\mathbb{R}^{n}\right)} \leq C Z_{\Omega}\|f\|_{\dot{F}_{p q}^{\alpha}\left(\mathbb{R}^{n}\right)}
$$

for all $f \in \mathcal{S}_{\infty}\left(\mathbb{R}^{n}\right)$.

(iii) Let $\alpha=0$. If $\Omega \in L \log L\left(S^{n-1}\right)$ and it satisfies the cancellation condition (1.2), then

$$
\left\|\mu_{\Omega, \rho, \phi, \alpha, q} f\right\|_{L^{p}\left(\mathbb{R}^{n}\right)} \leq C\|\Omega\|_{L \log L\left(S^{n-1}\right)}\|f\|_{\dot{F}_{p q}^{\alpha}\left(\mathbb{R}^{n}\right)}
$$

for all $f \in \mathcal{S}_{\infty}\left(\mathbb{R}^{n}\right)$.

In any case, by density we can extend (1.21), (1.24) and (1.25) and have them for all $f \in$ $\dot{F}_{p q}^{\alpha}\left(\mathbb{R}^{n}\right)$.

Note that (1.18) is referred to as the doubling condition. Thanks to the useful conversation with Professor XX Tao and Miss S He in the Zhejiang University of Science and Technology, we could improve our results.

We state our main result in full generality. Theorem 3 is almost a direct consequence of the next theorem.

Theorem 4 Suppose that we are given $\Omega \in L^{1}\left(S^{n-1}\right), \phi \in C^{1}\left(\mathbb{R}_{+}, \mathbb{R}_{+}\right)$and parameters $p, q$, $\alpha, \gamma, \rho$ satisfying

$$
1<p, q<\infty, \quad \rho>0, \quad \gamma>\frac{1}{2} \max \{\tilde{p}, \tilde{q}\},
$$

in addition to (1.18) and (1.19) in Theorem 3. Then: 
(i) Assume that

$$
\alpha \in\left(0, \frac{4}{c_{1} \log _{2} c_{0}} \cdot \frac{1 / \tilde{p}-1 /(2 \gamma)}{1-1 / \gamma} \cdot \frac{1 / \tilde{q}-1 /(2 \gamma)}{1-1 / \gamma}\right)
$$

If $b \in \Delta_{\gamma}\left(\mathbb{R}_{+}\right)$and $\Omega$ satisfies the cancellation condition (1.2), then

$$
\left\|\mu_{\Omega, \rho, \phi, \alpha, q}^{(b)} f\right\|_{L^{p}\left(\mathbb{R}^{n}\right)} \leq C\|\Omega\|_{L^{1}\left(S^{n-1}\right)}\|b\|_{\Delta_{\gamma}}\|f\|_{\dot{F}_{p q}^{\alpha}\left(\mathbb{R}^{n}\right)}
$$

for all $f \in \mathcal{S}_{\infty}\left(\mathbb{R}^{n}\right)$.

(ii) Assume $\alpha \in\left(-\min \left\{\frac{2 \beta}{c_{1} \log _{2} c_{0}} \cdot \frac{1 / \tilde{p}-1 /(2 \gamma)}{1-1 / \gamma} \cdot \frac{1 / \tilde{q}-1 /(2 \gamma)}{1-1 / \gamma}, \frac{\rho}{\log _{2} c_{0}}\right\}, 0\right)$ for some $\beta \in(0,1]$. If $b \in \Delta_{\max (\gamma, 2)}$ and

$$
W_{\Omega}:=\sup _{\xi^{\prime} \in S^{n-1}} \sqrt{\int_{S^{n-1} \times S^{n-1}} \frac{\left|\Omega\left(y^{\prime}\right) \Omega\left(z^{\prime}\right)\right|}{\left|\left(y^{\prime}-z^{\prime}\right) \cdot \xi^{\prime}\right|^{\beta}} d \sigma\left(y^{\prime}\right) d \sigma\left(z^{\prime}\right)}<+\infty,
$$

then

$$
\left\|\mu_{\Omega, \rho, \phi, \alpha, q}^{(b)} f\right\|_{L^{p}\left(\mathbb{R}^{n}\right)} \leq C W_{\Omega}\|b\|_{\Delta_{\max (\gamma, 2)}}\|f\|_{\dot{F}_{p q}^{\alpha}\left(\mathbb{R}^{n}\right)}
$$

for all $f \in \mathcal{S}_{\infty}\left(\mathbb{R}^{n}\right)$.

(iii) Assume $\alpha=0$. If $b \in \Delta_{\max (\gamma, 2)}, \Omega \in L \log L\left(S^{n-1}\right)$ and it satisfies the cancellation condition (1.2), then

$$
\left\|\mu_{\Omega, \rho, \phi, \alpha, q}^{(b)} f\right\|_{L^{p}\left(\mathbb{R}^{n}\right)} \leq C\|\Omega\|_{L \log L\left(S^{n-1}\right)}\|b\|_{\Delta_{\max (\gamma, 2)}}\|f\|_{\dot{F}_{p q}^{\alpha}\left(\mathbb{R}^{n}\right)}
$$

for all $f \in \mathcal{S}_{\infty}\left(\mathbb{R}^{n}\right)$.

In any case, by density we can extend (1.27), (1.29) and (1.30) and have them for all $f \in$ $\dot{F}_{p q}^{\alpha}\left(\mathbb{R}^{n}\right)$.

Theorem 3(i) and (iii) are direct consequences of Theorem 4. Indeed, assuming (1.20) and choosing $\gamma \gg 1$, we have (1.26). So, to obtain (i) we can apply Theorem 4 for such $\gamma$ with $b \equiv 1$. Theorem 3(iii) is a direct conseuqence of Theorem 4(iii). Note that in Theorems 3(ii) and 4(ii), the conditions of $\alpha$ is slightly improved.

Our strategy is to employ the Littlewood-Paley decomposition as Ding et al. did in [20]. However, we distort things via the sequence $\left\{a_{k}\right\}_{k \in \mathbb{Z}}$. We rely upon the modified Littlewood-Paley decomposition for the proof of Theorem 4, which we shall describe now. Let $\left\{a_{k}\right\}_{k \in \mathbb{Z}}$ be a lacunary sequence of positive numbers in the sense that $a_{k+1} / a_{k} \geq a>1$ $(k \in \mathbb{Z})$. A sequence $\left\{\Phi_{k}\right\}_{k \in \mathbb{Z}}$ of $C^{\infty}\left(\mathbb{R}^{n}\right)$-functions is said to be a partition of unity adapted to $\left\{a_{k}\right\}_{k \in \mathbb{Z}}$ if

$$
\begin{aligned}
& \operatorname{supp} \widehat{\Phi}_{k} \subset\left\{\xi \in \mathbb{R}^{n} ; a_{k-1} \leq|\xi| \leq a_{k+1}\right\} \quad(k \in \mathbb{Z}), \\
& \sum_{k \in \mathbb{Z}} \widehat{\Phi}_{k}(\xi)=1 \quad\left(\xi \in \mathbb{R}^{n} \backslash\{0\}\right),
\end{aligned}
$$

and

$$
\left|\xi^{\beta} \partial^{\beta} \widehat{\Phi}_{k}(\xi)\right| \leq C_{\beta}
$$

for any multiindex $\beta$. 
Denote by $\mathcal{P}$ the set of all polynomials in $\mathbb{R}^{n}$. Let $1<p, q<\infty$ and $\alpha \in \mathbb{R}$. For $f \in$

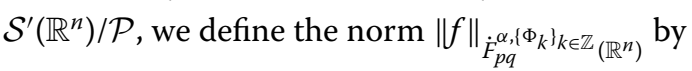

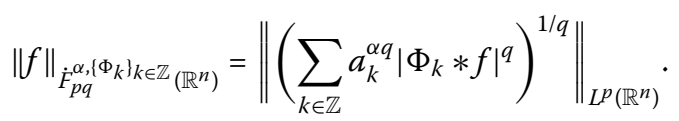

We admit that Proposition 1 below is true and we prove Theorem 4 first. We postpone the proof of Proposition 1 until the end of the paper.

Proposition 1 Let $\alpha \neq 0$ and $1<p, q<\infty$. Let $\left\{a_{k}\right\}_{k \in \mathbb{Z}}$ be a lacunary sequence of positive

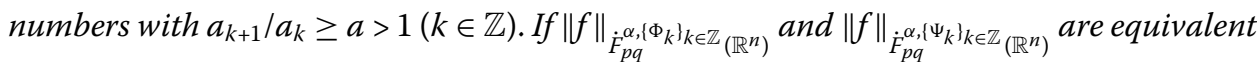
for any two partitions of unity, $\left\{\Phi_{k}\right\}_{k \in \mathbb{Z}}$ and $\left\{\Psi_{k}\right\}_{k \in \mathbb{Z}}$, adapted to $\left\{a_{k}\right\}_{k \in \mathbb{Z}}$, then there exists $C_{0}>$ a such that

$$
\frac{a_{k+1}}{a_{k}} \leq C_{0} \quad(k \in \mathbb{Z})
$$

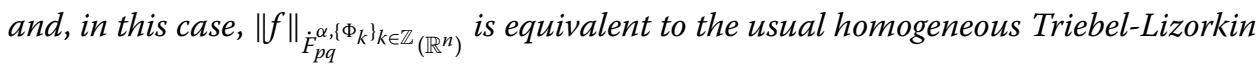
space norm $\|f\|_{\dot{F}_{p q}^{\alpha}\left(\mathbb{R}^{n}\right)}$.

In Sections 3-5, we shall prove Theorems 3 and 4 as well as Proposition 1, respectively.

\section{A strategy of the proof of Theorem 4}

\subsection{A setup}

For $t>0$, a function $b$ on $\mathbb{R}_{+}$and a homogeneous kernel $\Omega$ on $\mathbb{R}^{n}$, assume

$$
\int_{B(t) \backslash B(t / 2)}\left|b(|x|) \Omega\left(x^{\prime}\right)\right| d x<\infty .
$$

For $\rho>0$ and a nice function $\phi$, we define the family $\left\{\sigma_{t} ; t \in \mathbb{R}_{+}\right\}$of measures and the maximal operator $\sigma^{*}$ on $\mathbb{R}^{n}$ by

$$
\begin{aligned}
& \int_{\mathbb{R}^{n}} f(x) d \sigma_{t}(x)=\frac{1}{t^{\rho}} \int_{B(t) \backslash B(t / 2)} f\left(\phi(|x|) x^{\prime}\right) \frac{b(|x|) \Omega\left(x^{\prime}\right)}{|x|^{n-\rho}} d x, \\
& \sigma^{*} f(x)=\sup _{t>0}|| \sigma_{t}|* f(x)| \quad\left(x \in \mathbb{R}^{n}\right) .
\end{aligned}
$$

Note that the mapping $x \in \mathbb{R}^{n} \backslash\{0\} \mapsto \phi(|x|) x^{\prime} \in \mathbb{R}^{n} \backslash \overline{B(\inf \phi)}$ is a $C^{1}$-diffeomorphism, since $\phi \in C^{1}\left(\mathbb{R}_{+}, \mathbb{R}_{+}\right)$satisfies (1.18) and (1.19). Therefore, if we consider the measure $\sigma_{t}^{\dagger}$ by

$$
\int_{\mathbb{R}^{n}} f(x) d \sigma_{t}^{\dagger}(x)=\frac{1}{t^{\rho}} \int_{B(t) \backslash B(t / 2)} f(x) \frac{b(|x|) \Omega\left(x^{\prime}\right)}{|x|^{n-\rho}} d x
$$

then the above diffeomorphism induces $\sigma_{t}$. So, as regards the absolute value of $\sigma_{t}$, we have

$$
\int_{\mathbb{R}^{n}} f(x) d\left|\sigma_{t}\right|(x)=\frac{1}{t^{\rho}} \int_{B(t) \backslash B(t / 2)} f\left(\phi(|x|) x^{\prime}\right) \frac{\left|b(|x|) \Omega\left(x^{\prime}\right)\right|}{|x|^{n-\rho}} d x
$$


Denote by $\left\|\sigma_{t}\right\|$ the total mass of $\sigma_{t}$. A direct consequence of this alternative definition of $\left|\sigma_{t}\right|$ is that we have

$$
\left\|\sigma_{t}\right\| \leq C\|b\|_{\Delta_{1}}\|\Omega\|_{L^{1}}
$$

If we use (2.1), then we can write

$$
\tilde{\mu}_{\Omega, \alpha, \rho, q}^{(b)}(f)(x)=\left(\int_{0}^{\infty}\left|\sigma_{t} * f(x)\right|^{q} \frac{d t}{t \phi(t)^{q \alpha}}\right)^{1 / q} \quad\left(x \in \mathbb{R}^{n}\right) .
$$

Lemma 2.1 Let $\Omega \in L^{1}\left(S^{n-1}\right)$.

(1) For all admissible parameters,

$$
\left|\widehat{\sigma}_{t}(\xi)\right| \leq 2^{n-\rho}\|\Omega\|_{L^{1}\left(S^{n-1}\right)}\|b\|_{\Delta_{1}} \quad\left(t>0, \xi \in \mathbb{R}^{n}\right) .
$$

(2) If in addition $\Omega$ satisfies (1.2), then we have

$$
\left|\widehat{\sigma}_{t}(\xi)\right| \leq 2\|\Omega\|_{L^{1}\left(S^{n-1}\right)}\|b\|_{\Delta_{1}} \phi(t)|\xi| \quad\left(t>0, \xi \in \mathbb{R}^{n}\right) .
$$

Proof

(1) From the definition of the Fourier transform, we have an expression of $\widehat{\sigma}_{t}(\xi)$ :

$$
\widehat{\sigma}_{t}(\xi)=\frac{1}{t^{\rho}} \int_{B(t) \backslash B(t / 2)} \frac{b(|y|) \Omega\left(y^{\prime}\right)}{|y|^{n-\rho}} e^{-i \phi(|y|) y^{\prime} \cdot \xi} d y .
$$

From (2.7) we get (2.5).

(2) Using the cancellation property (1.2) of $\Omega$, we have another expression of $\widehat{\sigma}_{t}(\xi)$ :

$$
\widehat{\sigma}_{t}(\xi)=\frac{1}{t^{\rho}} \int_{B(t) \backslash B(t / 2)} \frac{b(|y|) \Omega\left(y^{\prime}\right)}{|y|^{n-\rho}}\left(e^{-i \phi(|y|) y^{\prime} \cdot \xi}-1\right) d y .
$$

From the monotonicity of $\phi$, (1.18) and (2.8) we obtain

$$
\begin{aligned}
\left|\widehat{\sigma}_{t}(\xi)\right| & \leq \frac{1}{t^{\rho}} \int_{t / 2}^{t}\left(\int_{S^{n-1}}\left|\Omega\left(y^{\prime}\right)\right| d \sigma\left(y^{\prime}\right)\right)|\xi| \cdot|\phi(r) b(r)| r^{\rho-1} d r \\
& \leq\|\Omega\|_{L^{1}\left(S^{n-1}\right)} \phi(t)|\xi| \int_{t / 2}^{t}|b(r)| \frac{d r}{r} \leq 2\|\Omega\|_{L^{1}\left(S^{n-1}\right)}\|b\|_{\Delta_{1}} \phi(t)|\xi| .
\end{aligned}
$$

So we are done.

As for the maximal operator $\sigma^{*}$ given by (2.2), we invoke the following lemma in [21, Lemma 3.2]: We define the directional Hardy-Littlewood maximal function of $F$ for a fixed vector $y^{\prime} \in S^{n-1}$ by

$$
M_{y^{\prime}} F(x)=\sup _{r>0} \frac{1}{2 r} \int_{-r}^{r}\left|f\left(x-t y^{\prime}\right)\right| d t .
$$

By the orthogonal decomposition $\mathbb{R}^{n}=H \oplus \mathbb{R} y^{\prime}$, we can prove that $M_{y^{\prime}}$ is bounded on $L^{p}\left(\mathbb{R}^{n}\right)$ for all $1<p<\infty$ and that the bound is uniform over $y^{\prime}$. By combining the Hölder inequality and the change of variables to polar coordinates, we can prove the following. 
Lemma 2.2 Let $\gamma>1$. Then there exists $C>0$ such that

$$
\sigma^{*}(f)(x) \leq C\|b\|_{\Delta_{\gamma}}\|\Omega\|_{L^{1}\left(S^{n-1}\right)}^{1 / \gamma}\left(\int_{S^{n-1}}\left|\Omega\left(y^{\prime}\right)\right| M_{y^{\prime}}\left(|f|^{\gamma^{\prime}}\right)(x) d \sigma\left(y^{\prime}\right)\right)^{1 / \gamma^{\prime}}
$$

for all $x \in \mathbb{R}^{n}$.

Thanks to Lemma 2.2 and the Minkowski inequality, for $p>\gamma^{\prime}$ there exists $C>0$ such that

$$
\left\|\sigma^{*}(f)\right\|_{L^{p}\left(\mathbb{R}^{n}\right)} \leq C\|b\|_{\Delta_{\gamma}}\|\Omega\|_{L^{1}\left(S^{n-1}\right)}\|f\|_{L^{p}\left(\mathbb{R}^{n}\right)} .
$$

From the monotonicity, (1.18) and (2.6) we get, for $\alpha \in \mathbb{R}, k \in \mathbb{Z}$,

$$
\left(\int_{2^{k}}^{2^{k+1}}\left|\widehat{\sigma}_{t}(\xi)\right|^{2} \frac{d t}{t \phi(t)^{2 \alpha}}\right)^{1 / 2} \leq 2\|\Omega\|_{L^{1}\left(S^{n-1}\right)}\|b\|_{\Delta_{1}}|\xi| \frac{\phi\left(2^{k}\right)}{\phi\left(2^{k}\right)^{\alpha}} .
$$

Using (1.18) and (1.19), we have the following.

Lemma 2.3 For any $0 \leq \beta<1$,

$$
\left|\widehat{\sigma}_{t}(\xi)\right| \leq C W_{\Omega}\|b\|_{\Delta_{2}} \frac{1}{(|\xi| \phi(t))^{\beta / 2}}
$$

for $\xi \in \mathbb{R}^{n}$. $W_{\Omega}$ is the quantity defined in (1.28).

For a precise proof, see the proof of [21, Lemma 2.4].

\subsection{Properties of $\phi$}

We denote $a_{j}:=1 / \phi\left(2^{-j}\right)$ and $a:=2^{1 /\|\varphi\|_{L^{\infty}}\left(\mathbb{R}_{+}\right)}>1$. Then $\left\{a_{j}\right\}_{j \in \mathbb{Z}}$ is also a lacunary sequence of the same lacunarity as $\left\{\phi\left(2^{j}\right)\right\}_{j \in \mathbb{Z}}$. From the assumption (1.18), it follows that

$$
\phi\left(2^{k} t\right) \leq c_{0}^{k} \phi(t)
$$

for $k \in \mathbb{N}$. It is easily seen from $(1.19)$ that $\left\{\phi\left(2^{j}\right)\right\}_{j \in \mathbb{Z}}$ is a lacunary sequence of positive numbers satisfying

$$
\phi\left(2^{k} t\right)=\phi(t) \exp \left(\int_{t}^{2^{k} t}(\log \phi(s))^{\prime} d s\right) \geq 2^{k /\|\varphi\|_{L} \infty_{\left(\mathbb{R}_{+}\right)} \phi(t)=a^{k} \phi(t)}
$$

for $k \in \mathbb{N}$ and $t>0$. See e.g. [21, Lemma 2.8] for details.

Note also that, for $\phi \in C^{1}\left(\mathbb{R}_{+}, \mathbb{R}_{+}\right)$satisfying (1.18), the condition (1.19) implies

$$
\phi(2 t) \geq C_{1} \phi(t) \quad(t>0)
$$

for some $C_{1}>1$. Indeed, assuming (1.18), there exists $s \in[t, 2 t]$

$$
\phi(2 t)-\phi(t)=t \phi^{\prime}(s) \geq c_{1} \frac{t}{s} \phi(s) \geq \frac{c_{1}}{c_{0}} \phi(t)
$$

by the mean value theorem, proving (2.15). 


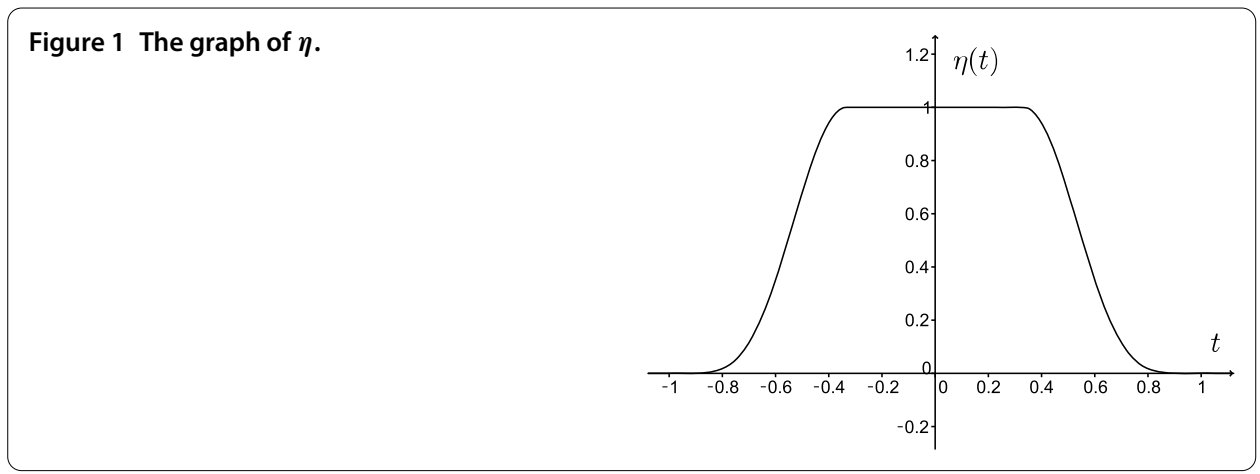

If in addition $\phi$ is concave, then (2.15) implies (1.19). Indeed,

$$
\phi^{\prime}(2 t) \geq \frac{\phi(2 t)-\phi(t)}{t} \geq\left(C_{1}-1\right) \frac{\phi(t)}{t} \quad(t>0) .
$$

\subsection{Construction of partition of unity}

For our purpose, we introduce a partition of unity and a characterization of the homogeneous Triebel-Lizorkin spaces associated to $\phi$ satisfying (1.18) and (1.19).

Take a nonincreasing $C^{\infty}(\mathbb{R})$-function $\eta$ such that $\chi_{[-1 / a, 1 / a]}(t) \leq \eta(t) \leq \chi_{[-1,1]}(t)$ for all $t \in \mathbb{R}$ (see Figure 1).

We define functions $\psi_{j}$ on $\mathbb{R}^{n}$ by

$$
\psi_{j}(\xi)=\eta\left(\frac{|\xi|}{a_{j+1}}\right)-\eta\left(\frac{|\xi|}{a_{j}}\right) \quad\left(\xi \in \mathbb{R}^{n}\right)
$$

Then observe that

$$
\psi_{j}(\xi)= \begin{cases}0, & 0 \leq|\xi| \leq a_{j} / a,|\xi| \geq a a_{j+1} \\ 1, & a a_{j} \leq t \leq a_{j+1}\end{cases}
$$

and that

$$
\begin{aligned}
& \operatorname{supp} \psi_{j} \subset\left\{a_{j} / a \leq|\xi| \leq a a_{j+1}\right\}, \\
& \operatorname{supp} \psi_{j} \cap \operatorname{supp} \psi_{\ell}=\emptyset, \quad \text { for }|j-\ell| \geq 2, \\
& \sum_{j \in \mathbb{Z}} \psi_{j}(\xi)=1 \quad\left(\mathbb{R}^{n} \backslash\{0\}\right) .
\end{aligned}
$$

That is, $\left\{\psi_{j}\right\}_{j \in \mathbb{Z}}$ is a smooth partition of unity adapted to $\left\{a_{j}\right\}_{j \in \mathbb{Z}}$.

Let $\Psi_{j}$ be defined on $\mathbb{R}^{n}$ by $\widehat{\Psi}_{j}(\xi)=\psi_{j}(\xi)$ for $\xi \in \mathbb{R}^{n}$. By Proposition 1 , we have

$$
\left\|\left(\sum_{j=-\infty}^{\infty}\left|a_{j}^{\alpha} \Psi_{j} * f\right|^{q}\right)^{1 / q}\right\|_{L^{p}} \approx\|f\|_{\dot{F}_{p q}^{\alpha}\left(\mathbb{R}^{n}\right)}
$$

if $a_{j+1} / a_{j} \leq b(j \in \mathbb{Z})$ for some $b \geq a$.

This condition is satisfied in our case, i.e. $a_{j+1} / a_{j}=\phi\left(2^{-j}\right) / \phi\left(2^{-j-1}\right) \leq c_{1}$. 


\subsection{A reduction by using the scaling invariance}

Now, using the definition of $\mu_{\Omega, \rho, \phi, \alpha, q}^{(b)}(f)(x)$ and the triangle inequality, via change of variables $y \mapsto 2^{k} y$, we obtain

$$
\begin{aligned}
& \mu_{\Omega, \rho, \phi, \alpha, q}^{(b)}(f)(x) \\
& \quad=\left(\int_{0}^{\infty}\left|\frac{1}{t^{\rho} \phi(t)^{\alpha}} \int_{B(t)} \frac{b(|y|) \Omega\left(y^{\prime}\right)}{|y|^{n-\rho}} f\left(x-\phi(|y|) y^{\prime}\right) d y\right|^{q} \frac{d t}{t}\right)^{1 / q} \\
& \quad=\left(\int_{0}^{\infty}\left|\sum_{k=0}^{\infty} \frac{1}{t^{\rho} \phi(t)^{\alpha}} \int_{B\left(2^{-k} t\right) \backslash B\left(2^{-k-1} t\right)} \frac{b(|y|) \Omega\left(y^{\prime}\right)}{|y|^{n-\rho}} f\left(x-\phi(|y|) y^{\prime}\right) d y\right|^{q} \frac{d t}{t}\right)^{1 / q} \\
& \quad \leq \sum_{k=0}^{\infty}\left(\int_{0}^{\infty}\left|\frac{1}{t^{\rho} \phi(t)^{\alpha}} \int_{B\left(2^{-k} t\right) \backslash B\left(2^{-k-1} t\right)} \frac{b(|y|) \Omega\left(y^{\prime}\right)}{|y|^{n-\rho}} f\left(x-\phi(|y|) y^{\prime}\right) d y\right|^{q} \frac{d t}{t}\right)^{1 / q} \\
& \quad=\sum_{k=0}^{\infty} \frac{1}{2^{\rho k}}\left(\int_{0}^{\infty}\left|\frac{1}{t^{\rho} \phi\left(2^{k} t\right)^{\alpha}} \int_{B(t) \backslash B(t / 2)} \frac{b\left(2^{k}|y|\right) \Omega\left(y^{\prime}\right)}{|y|^{n-\rho}} f\left(x-\phi\left(2^{k}|y|\right) y^{\prime}\right) d y\right|^{q} \frac{d t}{t}\right)^{1 / q} .
\end{aligned}
$$

Hence

$$
\begin{aligned}
& \mu_{\Omega, \rho, \phi, \alpha, q}^{(b)}(f)(x) \\
& \leq \sum_{k=0}^{\infty} \frac{1}{2^{\rho k}} \\
& \quad \times\left(\int_{0}^{\infty}\left|\frac{1}{t^{\rho} \phi\left(2^{k} t\right)^{\alpha}} \int_{B(t) \backslash B(t / 2)} \frac{b\left(2^{k}|y|\right) \Omega\left(y^{\prime}\right)}{|y|^{n-\rho}} f\left(x-\phi\left(2^{k}|y|\right) y^{\prime}\right) d y\right|^{q} \frac{d t}{t}\right)^{1 / q} .
\end{aligned}
$$

So, in the case $\alpha \geq 0$ we have

$$
\begin{aligned}
& \mu_{\Omega, \rho, \phi, \alpha, q}^{(b)}(f)(x) \\
& \leq \sum_{k=0}^{\infty} \frac{1}{2^{\left(\rho+\alpha /\|\varphi\|_{\infty}\right) k}} \\
& \quad \times\left(\int_{0}^{\infty}\left|\frac{1}{t^{\rho} \phi(t)^{\alpha}} \int_{B(t) \backslash B(t / 2)} \frac{b\left(2^{k}|y|\right) \Omega\left(y^{\prime}\right)}{|y|^{n-\rho}} f\left(x-\phi\left(2^{k}|y|\right) y^{\prime}\right) d y\right|^{q} \frac{d t}{t}\right)^{1 / q} .
\end{aligned}
$$

So, in the case $0>\alpha>-\rho / \log c_{0}$, from (2.22), we have

$$
\begin{aligned}
& \mu_{\Omega, \rho, \phi, \alpha, q}^{(b)}(f)(x) \\
& \leq \sum_{k=0}^{\infty} \frac{1}{2^{\left(\rho+\alpha \log _{2} c_{0}\right) k}} \\
& \quad \times\left(\int_{0}^{\infty}\left|\frac{1}{t^{\rho} \phi(t)^{\alpha}} \int_{B(t) \backslash B(t / 2)} \frac{b\left(2^{k}|y|\right) \Omega\left(y^{\prime}\right)}{|y|^{n-\rho}} f\left(x-\phi\left(2^{k}|y|\right) y^{\prime}\right) d y\right|^{q} \frac{d t}{t}\right)^{1 / q} .
\end{aligned}
$$

Notice that $b$ and $b\left(2^{k}\right.$.) satisfy the same condition due to the scaling invariance of $\Delta_{\gamma}$. Likewise $\phi$ and $\phi\left(2^{k}\right.$.) satisfy the same conditions (1.18) and (1.19) with constants independent of $k$. Hence, for our purpose, it is sufficient to consider the modified operator 
given by

$$
\tilde{\mu}_{\Omega, \rho, \phi, \alpha, q}^{(b)}(f)(x):=\left(\int_{0}^{\infty}\left|\frac{1}{t^{\rho} \phi(t)^{\alpha}} \int_{B(t) \backslash B(t / 2)} \frac{b(|y|) \Omega\left(y^{\prime}\right)}{|y|^{n-\rho}} f\left(x-\phi(|y|) y^{\prime}\right) d y\right|^{q} \frac{d t}{t}\right)^{1 / q}
$$

for $x \in \mathbb{R}^{n}$.

Now we proceed to the proof of Theorem 4. Let

$$
\tilde{\mu}_{\Omega, \rho, \phi, \alpha, q, f}^{(b)} f(x):=\left(\sum_{k=-\infty}^{\infty} \int_{2^{k}}^{2^{k+1}}\left|\Psi_{j-k} * \sigma_{t} * f(x)\right|^{q} \frac{d t}{t \phi(t)^{q \alpha}}\right)^{1 / q} \quad\left(x \in \mathbb{R}^{n}\right)
$$

for each $j$. Using the partition of unity (2.16) and the triangle inequality, we then have

$$
\begin{aligned}
\tilde{\mu}_{\Omega, \rho, \phi, \alpha, q}^{(b)} f(x) & =\left(\int_{0}^{\infty}\left|\sum_{j \in \mathbb{Z}} \Psi_{j} * \sigma_{t} * f(x)\right|^{q} \frac{d t}{t \phi(t)^{q \alpha}}\right)^{1 / q} \\
& =\left(\sum_{k \in \mathbb{Z}} \int_{2^{k}}^{2^{k+1}}\left|\sum_{j \in \mathbb{Z}} \Psi_{j-k} * \sigma_{t} * f(x)\right|^{q} \frac{d t}{t \phi(t)^{q \alpha}}\right)^{1 / q} \\
& \leq \sum_{j \in \mathbb{Z}}\left(\sum_{k \in \mathbb{Z}} \int_{2^{k}}^{2^{k+1}}\left|\Psi_{j-k} * \sigma_{t} * f(x)\right|^{q} \frac{d t}{t \phi(t)^{q \alpha}}\right)^{1 / q} \\
& \leq \sum_{j \in \mathbb{Z}} \tilde{\mu}_{\Omega, \rho, \phi, \alpha, q, j}^{(b)} f(x) .
\end{aligned}
$$

Next, we treat the $L^{p}$-estimate of $\tilde{\mu}_{\Omega, \rho, \phi, \alpha, q, j}^{(b)} f$.

Let us set

$$
\alpha(j):= \begin{cases}\alpha / c_{1}, & j \geq 0 \\ \alpha \log _{2} c_{0}, & j<0\end{cases}
$$

In Section 4 we plan to distinguish three cases to prove.

Lemma 2.4 Assume either one of the following three conditions:

1. $1<q<r<\gamma q<\infty$ (see Figure 2).

2. $1<q^{\prime}<r^{\prime}<\gamma q^{\prime}<\infty$ (see Figure 3).

3. $1<q=r<\infty$.

If $\Omega \in L^{1}\left(S^{n-1}\right)$, then we have

$$
\left\|\tilde{\mu}_{\Omega, \rho, \phi, \alpha, q, j}^{(b)} f\right\|_{L^{r}\left(\mathbb{R}^{n)}\right.} \leq C 2^{-\alpha(j) j}\|\Omega\|_{L^{1}\left(S^{n-1}\right)}\|f\|_{\dot{F}_{r q}^{\alpha}\left(\mathbb{R}^{n}\right)} .
$$

However, in case 3 , we just interpolate cases 1 and 2 . So we concentrate on cases 1 and 2 in Section 4.

Note that cases 1-3 do not cover all the cases as the above images show.

We also need to prove the following. 


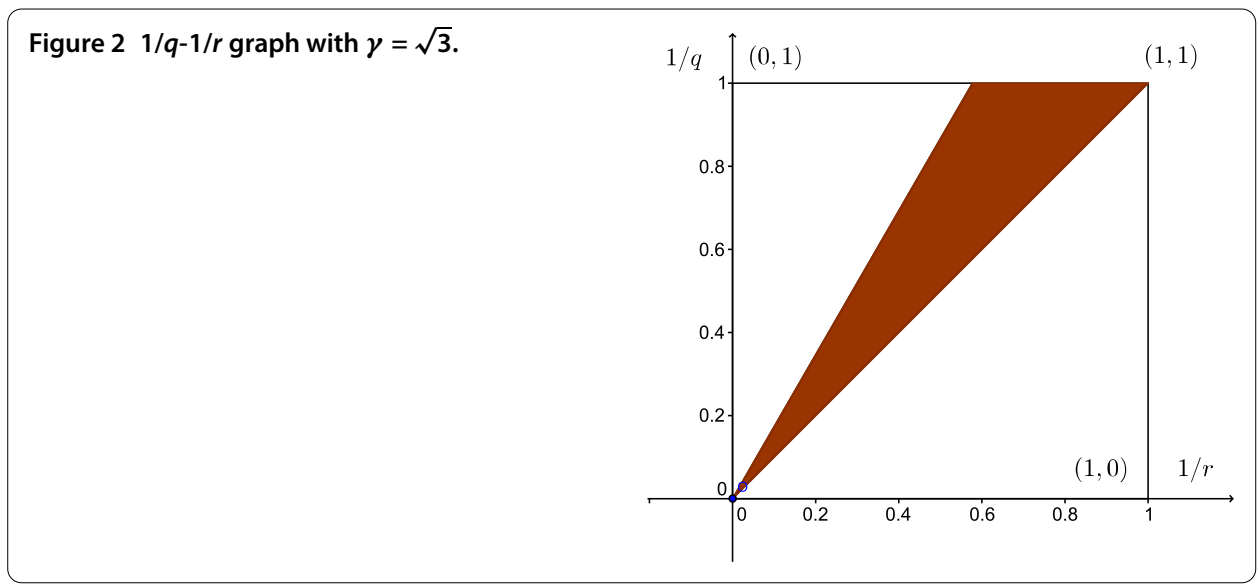

Figure $31 / q-1 / r$ graph with $\gamma=\sqrt{3}$.

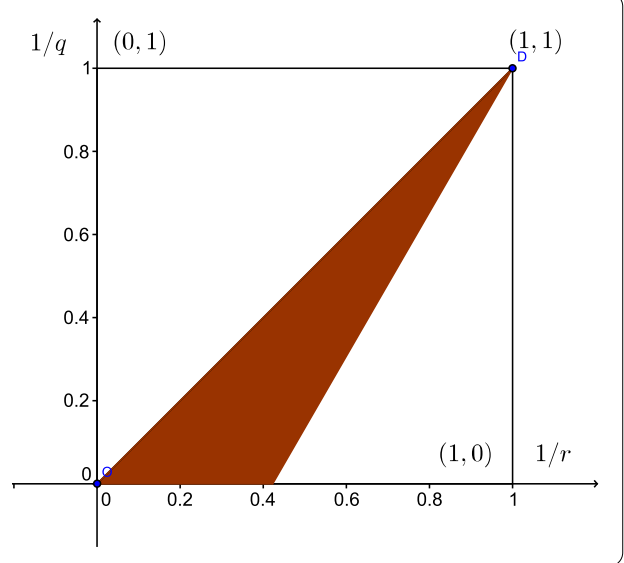

Lemma 2.5 Let $\phi$ satisfy the same conditions (1.18) and (1.19). Assume that $\Omega \in L^{1}\left(S^{n-1}\right)$ satisfies the cancellation condition (1.2). Then

$$
\left\|\tilde{\mu}_{\Omega, \rho, \phi, \alpha, 2, j}^{(b)} f\right\|_{L^{2}\left(\mathbb{R}^{n)}\right.} \leq C 2^{(\alpha(-j) / \alpha-\alpha(j)) j}\|\Omega\|_{L^{1}\left(S^{n-1}\right)}\|b\|_{\Delta_{\gamma}}\|f\|_{\dot{F}_{2,2}^{\alpha}} .
$$

By using the strong decay of (2.25), interpolate (2.25) and (2.26) to have (2.25) again for any admissible $p$ and $q$. Thus, in conclusion, (2.24) is summable over $j$ by virtue of (2.25).

\section{Proof of Theorem 4}

In this section, we prove Theorem 4 . One can obtain Theorem 4 by observing carefully the proof of [6, Theorem 6], but for the sake of completeness we shall give its detailed proof, modifying their one.

\subsection{Proof of Lemma 2.4}

Here we do not need the cancellation property of $\Omega$ and hence we can consider its absolute value of $\sigma_{t}$.

(1) In the case $q<r<\gamma q$, let

$$
J:=\left\|\tilde{\mu}_{\Omega, \rho, \phi, \alpha, q, j}^{(b)}\right\|_{L^{r}\left(\mathbb{R}^{n)}\right.}^{q}=\left\|\left(\sum_{k \in \mathbb{Z}} \int_{2^{k}}^{2^{k+1}}\left|\Psi_{j-k} * \sigma_{t} * f\right|^{q} \frac{d t}{t \phi(t)^{\alpha q}}\right)^{1 / q}\right\|_{L^{r}\left(\mathbb{R}^{n}\right)}^{q} .
$$


Let us set $s=(r / q)^{\prime}=r /(r-q)$. By the duality $L^{q / r}-L^{s}$, we can take a nonnegative function $h \in L^{s}\left(\mathbb{R}^{n}\right)$ with $\|h\|_{L^{s}\left(\mathbb{R}^{n}\right)}=1$ such that

$$
J=\int_{\mathbb{R}^{n}} \sum_{k \in \mathbb{Z}}\left\{\int_{2^{k}}^{2^{k+1}}\left|\Psi_{j-k} * \sigma_{t} * f(x)\right|^{q} \frac{d t}{t \phi(t)^{\alpha q}}\right\} h(x) d x .
$$

Denote by $\left\|\sigma_{t}\right\|$ the total mass of $\sigma_{t}$. By the Hölder inequality

$$
\begin{aligned}
J & =\sum_{k \in \mathbb{Z}} \int_{2^{k}}^{2^{k+1}}\left\{\int_{\mathbb{R}^{n}}\left|\int_{\mathbb{R}^{n}} \Psi_{j-k} * f(x-y) d \sigma_{t}(y)\right|^{q} h(x) d x\right\} \frac{d t}{t \phi(t)^{\alpha q}} \\
& \leq \sum_{k \in \mathbb{Z}} \int_{2^{k}}^{2^{k+1}}\left\{\int_{\mathbb{R}^{n}}\left[\int_{\mathbb{R}^{n}}\left|\Psi_{j-k} * f(x-y)\right|^{q} d\left|\sigma_{t}\right|(y)\right]\left\|\sigma_{t}\right\|^{q / q^{\prime}} h(x) d x\right\} \frac{d t}{t \phi(t)^{\alpha q}} .
\end{aligned}
$$

By virtue of (2.3), we have

$$
\begin{aligned}
J \leq & C\|\Omega\|_{L^{1}\left(S^{n-1}\right)}^{q}\|b\|_{\Delta_{1}} \\
& \times \sum_{k \in \mathbb{Z}} \int_{2^{k}}^{2^{k+1}}\left\{\int_{\mathbb{R}^{n}}\left[\int_{\mathbb{R}^{n}}\left|\Psi_{j-k} * f(y)\right|^{q} d\left|\sigma_{t}\right|(x-y)\right] h(x) d x\right\} \frac{d t}{t \phi(t)^{\alpha q}} \\
= & C\|\Omega\|_{L^{1}\left(S^{n-1}\right)}^{q}\|b\|_{\Delta_{1}} \\
& \times \sum_{k \in \mathbb{Z}} \int_{2^{k}}^{2^{k+1}}\left\{\int_{\mathbb{R}^{n}}\left|\Psi_{j-k} * f(y)\right|^{q}\left(\int_{\mathbb{R}^{n}} h(x) d\left|\sigma_{t}\right|(x-y)\right) d y\right\} \frac{d t}{t \phi(t)^{\alpha q}} .
\end{aligned}
$$

Since $1<q<r<\gamma q$, we have $s>\gamma^{\prime}$. So, by (2.10) and Hölder's inequality, we conclude

$$
\begin{aligned}
J^{1 / q} & \leq C\|\Omega\|_{L^{1}\left(S^{n-1}\right)}\|b\|_{\Delta_{\gamma}}\left(\int_{\mathbb{R}^{n}}\left(\sum_{k \in \mathbb{Z}} \int_{2^{k}}^{2^{k+1}}\left|\Psi_{j-k} * f(y)\right|^{q} \sigma^{*}(h)(y) \frac{d t}{t \phi(t)^{\alpha q}}\right) d y\right)^{1 / q} \\
& \leq C\|\Omega\|_{L^{1}\left(S^{n-1}\right)}\|b\|_{\Delta_{\gamma}}\left(\int_{\mathbb{R}^{n}} \sum_{k \in \mathbb{Z}} \frac{1}{\phi\left(2^{k}\right)^{\alpha q}}\left|\Psi_{j-k} * f(y)\right|^{q} \sigma^{*}(h)(y) d y\right)^{1 / q} \\
& \leq C\|\Omega\|_{L^{1}\left(S^{n-1}\right)}\|b\|_{\Delta_{\gamma}}\left(\int_{\mathbb{R}^{n}}\left(\sum_{k \in \mathbb{Z}} \frac{1}{\phi\left(2^{k}\right)^{\alpha q}}\left|\Psi_{j-k} * f(y)\right|^{q}\right)^{s^{\prime}} d y\right)^{1 /\left(s^{\prime} q\right)}\|h\|_{L^{s}\left(\mathbb{R}^{n}\right)}^{1 / q} \\
& =C\|\Omega\|_{L^{1}\left(S^{n-1}\right)}\|b\|_{\Delta_{\gamma}}\left(\int_{\mathbb{R}^{n}}\left(\sum_{\ell \in \mathbb{Z}} \frac{1}{\phi\left(2^{j-\ell}\right)^{\alpha q}}\left|\Psi_{\ell} * f(y)\right|^{q}\right)^{r / q} d y\right)^{1 / r} .
\end{aligned}
$$

Thus, we have

$$
\left\|\tilde{\mu}_{\Omega, \rho, \phi, \alpha, q, j}^{(b)} f\right\|_{L^{r}\left(\mathbb{R}^{n)}\right.} \leq C 2^{-\alpha(j) j}\|b\|_{\Delta_{\gamma}}\|f\|_{\dot{F}_{r q}^{\alpha}} .
$$

(2) In case $1<r<q$ and $r^{\prime}<\gamma q^{\prime}$, it follows that $r^{\prime}>q^{\prime}$. By duality, there is a sequence of functions $g_{k}(x, t)$ such that

$$
\left(\int_{\mathbb{R}^{n}}\left(\sum_{k \in \mathbb{Z}} \int_{2^{k}}^{2^{k+1}}\left|g_{k}(x, t)\right|^{q^{\prime}} \frac{d t}{t}\right)^{r^{\prime} \mid q^{\prime}} d x\right)^{1 / r^{\prime}}=1
$$


Sawano and Yabuta Journal of Inequalities and Applications 2014, 2014:232

Page 16 of 29

http://www.journalofinequalitiesandapplications.com/content/2014/1/232

and such that

$$
\begin{aligned}
& \left\|\left(\sum_{k \in \mathbb{Z}} \int_{2^{k}}^{2^{k+1}}\left|\Psi_{j-k} * \sigma_{t} * f\right|^{q} \frac{d t}{t \phi(t)^{\alpha q}}\right)^{1 / q}\right\|_{L^{r}\left(\mathbb{R}^{n}\right)} \\
& \quad=\int_{\mathbb{R}^{n}} \sum_{k \in \mathbb{Z}}\left\{\int_{2^{k}}^{2^{k+1}}\left(\Psi_{j-k} * \sigma_{t} * f(x)\right) g_{k}(x, t) \frac{d t}{t \phi(t)^{\alpha}}\right\} d x .
\end{aligned}
$$

Then we have

$$
\begin{aligned}
& \int_{\mathbb{R}^{n}} \sum_{k \in \mathbb{Z}} \int_{2^{k}}^{2^{k+1}}\left(\Psi_{j-k} * \sigma_{t} * f(x)\right) g_{k}(x, t) \frac{d t}{t \phi(t)^{\alpha}} d x \\
& \leq \int_{\mathbb{R}^{n}} \sum_{k \in \mathbb{Z}}\left\{\int_{2^{k}}^{2^{k+1}}\left(\int_{\mathbb{R}^{n}}\left|\Psi_{j-k} * f(y)\right| d\left|\sigma_{t}\right|(x-y)\right)\left|g_{k}(x, t)\right| \frac{d t}{t \phi(t)^{\alpha}}\right\} d x \\
& \leq \int_{\mathbb{R}^{n}} \sum_{k \in \mathbb{Z}}\left\{\int_{2^{k}}^{2^{k+1}}\left|\Psi_{j-k} * f(y)\right|\left(\int_{\mathbb{R}^{n}}\left|g_{k}(x, t)\right| d\left|\sigma_{t}\right|(x-y)\right) \frac{d t}{t \phi(t)^{\alpha}}\right\} d x \\
& \quad \leq C \int_{\mathbb{R}^{n}} \sum_{k \in \mathbb{Z}}\left\{\int_{2^{k}}^{2^{k+1}} \frac{1}{\phi\left(2^{k}\right)^{\alpha}}\left|\Psi_{j-k} * f(y)\right|\left(\int_{\mathbb{R}^{n}}\left|g_{k}(x, t)\right| d\left|\sigma_{t}\right|(x-y)\right) \frac{d t}{t}\right\} d y .
\end{aligned}
$$

By using the Hölder inequality for sequences, we have

$$
\begin{aligned}
\int_{\mathbb{R}^{n}} & \left(\sum_{k \in \mathbb{Z}} \int_{2^{k}}^{2^{k+1}}\left(\Psi_{j-k} * \sigma_{t} * f(x)\right) g_{k}(x, t) \frac{d t}{t \phi(t)^{\alpha}}\right) d x \\
\leq & C \int_{\mathbb{R}^{n}}\left(\sum_{k \in \mathbb{Z}} \frac{1}{\phi\left(2^{k}\right)^{\alpha q}}\left|\Psi_{j-k} * f(y)\right|^{q}\right)^{1 / q} \\
& \times\left(\sum_{k \in \mathbb{Z}} \int_{2^{k}}^{2^{k+1}}\left(\int_{\mathbb{R}^{n}}\left|g_{k}(x, t)\right| d\left|\sigma_{t}\right|(x-y)\right)^{q^{\prime}} \frac{d t}{t}\right)^{1 / q^{\prime}} d y .
\end{aligned}
$$

By the properties of $\phi$ and Proposition 1, we conclude

$$
\begin{aligned}
\int_{\mathbb{R}^{n}} & \left(\sum_{k \in \mathbb{Z}} \int_{2^{k}}^{2^{k+1}}\left(\Psi_{j-k} * \sigma_{t} * f(x)\right) g_{k}(x, t) \frac{d t}{t \phi(t)^{\alpha}}\right) d x \\
\leq & C 2^{-\alpha(j) j}\|b\|_{\Delta_{\gamma}}\left(\int_{\mathbb{R}^{n}}\left(\sum_{k \in \mathbb{Z}} \frac{1}{\phi\left(2^{k}\right)^{\alpha q}}\left|\Psi_{k} * f(y)\right|^{q}\right)^{r / q} d y\right)^{1 / r} \\
& \times\left(\int_{\mathbb{R}^{n}}\left(\sum_{k \in \mathbb{Z}} \int_{2^{k}}^{2^{k+1}}\left(\int_{\mathbb{R}^{n}}\left|g_{k}(x, t)\right| d\left|\sigma_{t}\right|(x-y)\right)^{q^{\prime}} \frac{d t}{t}\right)^{r^{\prime} / q^{\prime}} d y\right)^{1 / r^{\prime}} \\
= & C 2^{-\alpha(j) j}\|b\|_{\Delta_{\gamma}}\|f\|_{\dot{F}_{r q}^{\alpha}} \\
& \times\left(\int_{\mathbb{R}^{n}}\left(\sum_{k \in \mathbb{Z}} \int_{2^{k}}^{2^{k+1}}\left(\int_{\mathbb{R}^{n}}\left|g_{k}(x, t)\right| d\left|\sigma_{t}\right|(x-y)\right)^{q^{\prime}} \frac{d t}{t}\right)^{r^{\prime} / q^{\prime}} d y\right)^{1 / r^{\prime}} .
\end{aligned}
$$


In the same way as in [6, p.705], using (2.10), we can check

$$
\begin{gathered}
\left(\int_{\mathbb{R}^{n}}\left(\sum_{k \in \mathbb{Z}} \int_{2^{k}}^{2^{k+1}}\left(\int_{\mathbb{R}^{n}}\left|g_{k}(x, t)\right| d\left|\sigma_{t}\right|(x-y)\right)^{q^{\prime}} \frac{d t}{t}\right)^{r^{\prime} / q^{\prime}} d y\right)^{1 / r^{\prime}} \\
\leq C\|\Omega\|_{L^{1}\left(S^{n-1}\right)}\left(\int_{\mathbb{R}^{n}}\left(\sum_{k \in \mathbb{Z}} \int_{2^{k}}^{2^{k+1}}\left|g_{k}(x, t)\right|^{q^{\prime}} \frac{d t}{t}\right)^{r^{\prime} / q^{\prime}} d x\right)^{1 / r^{\prime}},
\end{gathered}
$$

if $\left(\frac{r^{\prime}}{q^{\prime}}\right)^{\prime}>\gamma^{\prime}$. Hence we have, for $1<q^{\prime}<r^{\prime}<\gamma q^{\prime}$,

$$
\left\|\tilde{\mu}_{\Omega, \rho, \phi, \alpha, q, j}^{(b)} f\right\|_{L^{r}\left(\mathbb{R}^{n}\right)} \leq C 2^{-\alpha(j) j}\|f\|_{\dot{F}_{r q}^{\alpha}} .
$$

So we are done.

\subsection{Proof of Lemma 2.5}

By virtue of the Plancherel theorem and the Fubini theorem, we have

$$
\begin{aligned}
\left\|\tilde{\mu}_{\Omega, \rho, \phi, \alpha, 2, j}^{(b)} f\right\|_{L^{2}}^{2} & =\sum_{k=-\infty}^{\infty} \int_{\mathbb{R}^{n}}\left(\int_{2^{k}}^{2^{k+1}}\left|\Psi_{j-k} * \sigma_{t} * f(x)\right|^{2} \frac{d t}{t \phi(t)^{2 \alpha}}\right) d x \\
& =C \sum_{k=-\infty}^{\infty} \int_{\mathbb{R}^{n}}\left(\int_{2^{k}}^{2^{k+1}}\left|\widehat{\sigma}_{t}(\xi)\right|^{2} \frac{d t}{t \phi(t)^{2 \alpha}}\right)|\hat{f}(\xi)|^{2} \psi_{j-k}(\xi)^{2} d \xi .
\end{aligned}
$$

By (2.11), (3.3) and the support property of $\psi_{j-k}$, we have

$$
\begin{aligned}
\left\|\tilde{\mu}_{\Omega, \rho, \phi, \alpha, 2, j}^{(b)} f\right\|_{L^{2}}^{2} & \leq C\|b\|_{\Delta_{1}} \sum_{k=-\infty}^{\infty} \int_{\mathbb{R}^{n}} \frac{|\xi|^{2}}{\phi\left(2^{k}\right)^{2 \alpha-2}}|\hat{f}(\xi)|^{2} \psi_{j-k}(\xi)^{2} d \xi \\
& \leq C\|b\|_{\Delta_{1}} \sum_{\ell=-\infty}^{\infty} \int_{\mathbb{R}^{n}} \frac{1}{\phi\left(2^{-\ell}\right)^{2} \phi\left(2^{j-\ell}\right)^{2 \alpha-2}}|\hat{f}(\xi)|^{2} \psi_{\ell}(\xi)^{2} d \xi .
\end{aligned}
$$

For $j \leq 0$, from (2.14), it follows that $\phi\left(2^{j-\ell}\right) \leq C 2^{j / c_{1}} \phi\left(2^{-\ell}\right)$, and for $j \geq 0$, from (2.13) we get $\phi\left(2^{j-\ell}\right) \leq 2^{j \log _{2} c_{0}} \phi\left(2^{-\ell}\right)$. Likewise, we have $\phi\left(2^{-\ell}\right) \leq 2^{-\alpha(j) j} \phi\left(2^{j-\ell}\right)$.

We need to control the integrand; first of all,

$$
\frac{1}{\phi\left(2^{j-l}\right)^{2 \alpha-2}}=\frac{\phi\left(2^{j-l}\right)^{2}}{\phi\left(2^{j-l}\right)^{2 \alpha}} .
$$

When $j \geq 0$, we use

$$
\phi\left(2^{j-\ell}\right)^{2} \leq C 2^{2 j \log _{2} c_{0}} \phi\left(2^{-\ell}\right)^{2}
$$

and

$$
\left(\frac{1}{\phi\left(2^{j-\ell}\right)}\right)^{2 \alpha} \leq C\left(\frac{2^{-\alpha(j) j}}{\phi\left(2^{-\ell}\right)}\right)^{2 \alpha} \leq C \frac{2^{-2\left(\alpha / c_{1}\right) j}}{\phi\left(2^{-\ell}\right)^{2 \alpha}}
$$

When $j \leq 0$, we use

$$
\phi\left(2^{j-\ell}\right)^{2} \leq C 2^{2 j / c_{1}} \phi\left(2^{-\ell}\right)^{2}
$$


and

$$
\left(\frac{1}{\phi\left(2^{j-\ell}\right)}\right)^{2 \alpha} \leq C\left(\frac{2^{-\alpha(j) j}}{\phi\left(2^{-\ell}\right)}\right)^{2 \alpha} \leq C \frac{2^{-2 \alpha\left(\log _{2} c_{0}\right) j}}{\phi\left(2^{-\ell}\right)^{2 \alpha}} .
$$

So, if $j \geq 0$, we have

$$
\begin{aligned}
\left\|\tilde{\mu}_{\Omega, \rho, \phi, \alpha, 2, j}^{(b)} f\right\|_{L^{2}}^{2} & \leq C 2^{2\left(\log _{2} c_{0}-\alpha / c_{1}\right) j} \sum_{\ell=-\infty}^{\infty} \int_{\mathbb{R}^{n}} \frac{1}{\phi\left(2^{-\ell}\right)^{2 \alpha}}|\hat{f}(\xi)|^{2} \psi_{\ell}(\xi)^{2} d \xi \\
& \leq C 2^{2\left(\log _{2} c_{0}-\alpha / c_{1}\right) j}\|f\|_{\dot{F}_{2,2}^{\alpha}}^{2} .
\end{aligned}
$$

Hence, after incorporating a similar estimate for $j \leq 0$, we get (2.26).

\subsection{Interpolation and the conclusion of the proof of (i)}

Let

$$
\alpha \in\left(0, \frac{1}{c_{1} \log _{2} c_{0}} \cdot \frac{1 / \tilde{p}-1 /(2 \gamma)}{1 / 2-1 /(2 \gamma)} \cdot \frac{1 / \tilde{q}-1 /(2 \gamma)}{1 / 2-1 /(2 \gamma)}\right) .
$$

By interpolating (2.26) and (2.25), we claim that there exists $\delta>0$ such that

$$
\left\|\tilde{\mu}_{\Omega, \rho, \phi, \alpha, q, j}^{(b)} f\right\|_{L^{p}\left(\mathbb{R}^{n}\right)} \leq C 2^{-\delta|j|}\|f\|_{\dot{F}_{p q}^{\alpha}} .
$$

When $p=q=2$, then (3.4) is correct by virtue of $(2.25)(j \geq 0)$ and $(2.26)(j<0)$. We check next the case $p \neq 2$ and $q \neq 2$. For $j \geq 0$, by (2.25) we may take $\delta=\alpha(j)=\alpha / c_{1}$. For $j \leq-1$, we take $1<r_{1}, r_{2}<\infty$ and $0<\theta_{1}, \theta_{2}<1$ satisfying

$$
\begin{aligned}
& \frac{1}{p}=\frac{\theta_{1}}{2}+\frac{1-\theta_{1}}{r_{1}}, \\
& \frac{1}{q}=\frac{\theta_{2}}{2}+\frac{1-\theta_{2}}{r_{2}} .
\end{aligned}
$$

Note that we have

$$
(p-2)\left(r_{1}-2\right)>0, \quad(q-2)\left(r_{2}-2\right)>0 .
$$

We choose $1<r_{1}, r_{2}<\infty$ so that

$$
\tilde{p}<\tilde{r_{1}}<2 \gamma, \quad \tilde{q}<\tilde{r_{2}}<2 \gamma
$$

and then determine $\theta_{1}, \theta_{2}$ by (3.5), (3.6). As in the Figure 4, we can arrange that

$$
\alpha<\frac{\theta_{1} \theta_{2}}{c_{1} \log _{2} c_{0}}<\frac{1}{c_{1} \log _{2} c_{0}} \cdot \frac{1 / \tilde{p}-1 /(2 \gamma)}{1 / 2-1 /(2 \gamma)} \cdot \frac{1 / \tilde{q}-1 /(2 \gamma)}{1 / 2-1 /(2 \gamma)} .
$$

We shall see that this choice is possible. Recall that $\tilde{p}, \tilde{q}<2 \gamma$. Then some arithmetic shows that

$$
\theta_{1}=\frac{1 / p-1 / r_{1}}{1 / 2-1 / r_{1}}=\frac{1 / p^{\prime}-1 / r_{1}^{\prime}}{1 / 2-1 / r_{1}^{\prime}}=\frac{1 / \tilde{p}-1 / \tilde{r}_{1}}{1 / 2-1 / \tilde{r}_{1}}
$$




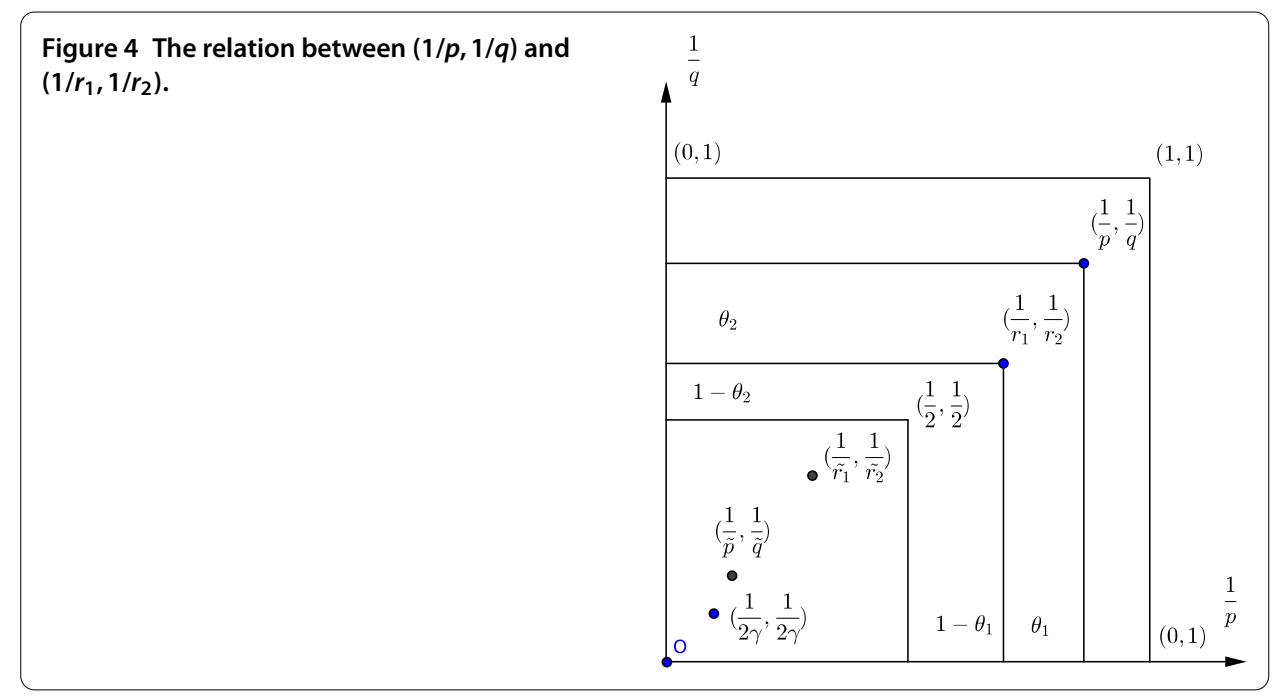

and that

$$
\theta_{2}=\frac{1 / q-1 / r_{2}}{1 / 2-1 / r_{2}}=\frac{1 / q^{\prime}-1 / r_{2}^{\prime}}{1 / 2-1 / r_{2}^{\prime}}=\frac{1 / \tilde{q}-1 / \tilde{r_{2}}}{1 / 2-1 / \tilde{r_{2}}}
$$

Assuming that $\tilde{p}, \tilde{q}>2$, we conclude that the parameters $\theta_{1}$ and $\theta_{2}$ are increasing on $(2, \infty)$ with respect to $\tilde{r_{1}}$ and $\tilde{r_{2}}$ as functions in $\tilde{r_{1}}$ and $\tilde{r_{2}}$, respectively. Hence

$$
\theta_{1} \theta_{2}=\frac{1 / \tilde{p}-1 / \tilde{r_{1}}}{1 / 2-1 / \tilde{r_{1}}} \cdot \frac{1 / \tilde{q}-1 / \tilde{r_{2}}}{1 / 2-1 / \tilde{r_{2}}}<\frac{1 / \tilde{p}-1 /(2 \gamma)}{1 / 2-1 /(2 \gamma)} \cdot \frac{1 / \tilde{q}-1 /(2 \gamma)}{1 / 2-1 /(2 \gamma)} .
$$

Therefore, since

$$
0<\alpha<\frac{1}{c_{1} \log _{2} c_{0}} \cdot \frac{1 / \tilde{p}-1 /(2 \gamma)}{1 / 2-1 /(2 \gamma)} \cdot \frac{1 / \tilde{q}-1 /(2 \gamma)}{1 / 2-1 /(2 \gamma)},
$$

and $\tilde{p}, \tilde{q}<2 \gamma$, we get (3.7) by choosing $r_{1}$ sufficiently near $2 \gamma$ if $p>2$ and $r_{1}^{\prime}$ sufficiently near $2 \gamma$ if $1<p<2$, and by choosing $r_{2}$ similarly according to $q>2$ or $1<q<2$.

Now, interpolating (2.26) and (2.25) with $r=r_{1}, q=2$, we get

$$
\left\|\tilde{\mu}_{\Omega, \rho, \phi, \alpha, 2, j}^{(b)} f\right\|_{L^{p}\left(\mathbb{R}^{n}\right)} \leq C 2^{\left(\theta_{1}\left(1 / c_{1}-\alpha \log _{2} c_{0}\right)-\left(1-\theta_{1}\right) \alpha \log _{2} c_{0}\right) j}\|f\|_{\dot{F}_{p 2}^{\alpha}} .
$$

We then interpolate (2.25) and (3.8) with $r=p, q=r_{2}$. As a consequence, we have

$$
\left\|\tilde{\mu}_{\Omega, \rho, \phi, \alpha, q, j}^{(b)} f\right\|_{L^{p}\left(\mathbb{R}^{n}\right)} \leq C 2^{\left\{\theta_{2}\left(\theta_{1}\left(1 / c_{1}-\alpha \log _{2} c_{0}\right)-\left(1-\theta_{1}\right) \alpha \log _{2} c_{0}\right)-\left(1-\theta_{2}\right) \alpha \log _{2} c_{0}\right\} j}\|f\|_{\dot{F}_{p q}^{\alpha}} .
$$

An arithmetic together with (3.7) shows that

$$
\theta_{2}\left(\theta_{1}\left(1 / c_{1}-\alpha \log _{2} c_{0}\right)-\left(1-\theta_{1}\right) \alpha \log _{2} c_{0}\right)-\left(1-\theta_{2}\right) \alpha \log _{2} c_{0}=\theta_{1} \theta_{2} / c_{1}-\alpha \log _{2} c_{0}>0 .
$$

Thus, taking $\delta=\min \left\{\alpha / c_{1}, \theta_{1} \theta_{2} / c_{1}-\alpha \log _{2} c_{0}\right\}$, we obtain the desired estimate (3.4).

In the case $p=2$ or $q=2$, we can get the desired estimate more simply, by applying interpolation once. 
Thus by (2.24) and (3.4) we obtain

$$
\left\|\tilde{\mu}_{\Omega, \rho, \phi, \alpha, q}^{(b)} f\right\|_{L^{p}\left(\mathbb{R}^{n)}\right.} \leq \sum_{j \in \mathbb{Z}}\left\|\tilde{\mu}_{\Omega, \rho, \phi, \alpha, q, j}^{(b)} f\right\|_{L^{p}\left(\mathbb{R}^{n}\right)} \leq C\|f\|_{\dot{F}_{p q}^{\alpha}} .
$$

This completes the proof of Theorem 4(i).

\subsection{The proof of (ii)}

Below we shall prove Theorem 4(ii). By the Schwarz inequality, we have

$$
\begin{aligned}
\left|\widehat{\sigma}_{t}(\xi)\right| & =\frac{1}{t^{\rho}}\left|\int_{t / 2<|y| \leq t} e^{-i \phi(|y|) y^{\prime} \cdot \xi} \frac{b(|y|) \Omega\left(y^{\prime}\right)}{|y|^{n-\rho}} d y\right| \\
& =\frac{1}{t^{\rho}}\left|\int_{t / 2}^{t}\left(\int_{S^{n-1}} \Omega\left(y^{\prime}\right) e^{-i \phi(r) y^{\prime} \cdot \xi} d \sigma\left(y^{\prime}\right)\right) b(r) r^{\rho-1} d r\right| \\
& \leq\left(\int_{t / 2}^{t}|b(r)|^{2} \frac{d r}{r}\right)^{1 / 2}\left(\int_{t / 2}^{t}\left|\int_{S^{n-1}} \Omega\left(y^{\prime}\right) e^{-i \phi(r) y^{\prime} \cdot \xi} d \sigma\left(y^{\prime}\right)\right|^{2} \frac{d r}{r}\right)^{1 / 2} \\
& \leq C\|b\|_{\Delta_{2}}\left(\int_{t / 2}^{t}\left|\int_{S^{n-1}} \Omega\left(y^{\prime}\right) e^{-i \phi(r) y^{\prime} \cdot \xi} d \sigma\left(y^{\prime}\right)\right|^{2} \frac{d r}{r}\right)^{1 / 2} .
\end{aligned}
$$

Recall

$$
W_{\Omega}=\sup _{\xi \in \mathbb{R}^{n} \backslash\{0\}}\left(\int_{S^{n-1} \times S^{n-1}} \frac{\left|\Omega\left(y^{\prime}\right) \Omega\left(z^{\prime}\right)\right|}{\left|\left(y^{\prime}-z^{\prime}\right) \cdot \xi^{\prime}\right|^{\beta}} d \sigma\left(y^{\prime}\right) d \sigma\left(z^{\prime}\right)\right)^{1 / 2} .
$$

Then, by (2.12) and the doubling condition of $\phi$, we have

$$
\left(\int_{2^{k}}^{2^{k+1}}\left|\widehat{\sigma}_{t}(\xi)\right|^{2} \frac{d t}{t \phi(t)^{2 \alpha}}\right)^{1 / 2} \leq \frac{C W_{\Omega}\|b\|_{\Delta_{2}}}{|\xi|^{\beta / 2} \phi\left(2^{k}\right)^{\beta / 2} \phi\left(2^{k}\right)^{\alpha}} .
$$

By (3.3), (3.11) and the support property of $\psi_{j-k}$, we have

$$
\begin{aligned}
\left\|\tilde{\mu}_{\Omega, \rho, \phi, \alpha, 2, j}^{(b)} f\right\|_{L^{2}\left(\mathbb{R}^{n}\right)}^{2} & \leq C W_{\Omega}^{2}\|b\|_{\Delta_{2}}^{2} \sum_{k=-\infty}^{\infty} \int_{\mathbb{R}^{n}} \frac{|\hat{f}(\xi)|^{2} \psi_{j-k}(|\xi|)^{2}}{|\xi|^{\beta} \phi\left(2^{k-1}\right)^{\beta} \phi\left(2^{k+1}\right)^{2 \alpha}} d \xi \\
& \leq C W_{\Omega}^{2}\|b\|_{\Delta_{2}}^{2} \sum_{\ell=-\infty}^{\infty} \int_{\mathbb{R}^{n}}\left(\frac{\phi\left(2^{-\ell}\right)}{\phi\left(2^{j-\ell}\right)}\right)^{\beta} \phi\left(2^{j-\ell}\right)^{-2 \alpha}|\hat{f}(\xi)|^{2} \psi_{\ell}(|\xi|)^{2} d \xi .
\end{aligned}
$$

As in the case (i), we have

$$
\phi\left(2^{-\ell}\right) \leq 2^{-j / c_{1}} \phi\left(2^{j-\ell}\right)
$$

for $j \geq 0$, and

$$
\phi\left(2^{-\ell}\right) \leq 2^{-j \log _{2} c_{0}} \phi\left(2^{j-\ell}\right)
$$

for $j \leq 0$. Similarly, we have

$$
\phi\left(2^{j-\ell}\right) \leq 2^{j / c_{1}} \phi\left(2^{-\ell}\right)
$$


for $j \leq 0$ and

$$
\phi\left(2^{j-\ell}\right) \leq c_{0}^{j-1}=2^{j \log _{2} c_{0}} \phi\left(2^{-\ell}\right)
$$

for $j \geq 0$. So, as in the $L^{2}$-estimate in (i), we obtain

$$
\left\|\tilde{\mu}_{\Omega, \rho, \phi, \alpha, 2, f}^{(b)} f\right\|_{L^{2}\left(\mathbb{R}^{n}\right)} \leq \begin{cases}C 2^{-\left(\beta /\left(2 c_{1}\right)+\alpha \log _{2} c_{0}\right) j} W_{\Omega}\|b\|_{\Delta_{2}}\|f\|_{\dot{F}_{2,2}^{\alpha}} & \text { if } j \geq 1, \\ C 2^{-\left(\left(\beta \log _{2} c_{0}\right) / 2+\alpha / c_{1}\right) j} W_{\Omega}\|b\|_{\Delta_{2}}\|f\|_{\dot{F}_{2,2}^{\alpha}}^{\alpha} & \text { if } j \leq 0 .\end{cases}
$$

As for the $L^{p}$-estimate, since $\alpha<0$, we use $\phi\left(2^{j-\ell}\right) \leq c_{0}^{j} \phi\left(2^{-\ell}\right)$ for $j \geq 0$ and $\phi\left(2^{j-\ell}\right) \leq$ $2^{j / c_{1}} \phi\left(2^{-\ell}\right)$ for $j \leq 0$. Hence we get, as in the $L^{p}$-estimate in (i), for any $1<q, r<\infty$ with $\tilde{r}<\gamma \tilde{q}$ and $j \in \mathbb{Z}$

$$
\left\|\tilde{\mu}_{\Omega, \rho, \phi, \alpha, q, j}^{(b)} f\right\|_{L^{r}\left(\mathbb{R}^{n}\right)} \leq \begin{cases}C 2^{-\left(\alpha / c_{1}\right) j} W_{\Omega}\|b\|_{\Delta_{2}}\|f\|_{\dot{F}_{r q}^{\alpha}}^{\alpha} & \text { for } j \leq 0, \\ C 2^{-\left(\alpha \log _{2} c_{0}\right) j} W_{\Omega}\|b\|_{\Delta_{2}}\|f\|_{\dot{F}_{r q}^{\alpha}}^{\alpha} & \text { for } j \geq 0 .\end{cases}
$$

It follows that, for

$$
\alpha \in\left(-\frac{2 \beta}{c_{1} \log _{2} c_{0}} \cdot \frac{1 / \tilde{p}-1 /(2 \gamma)}{1-1 / \gamma} \cdot \frac{1 / \tilde{q}-1 /(2 \gamma)}{1-1 / \gamma}, 0\right)
$$

there still exists $\delta>0$ such that

$$
\left\|\tilde{\mu}_{\Omega, \rho, \phi, \alpha, q, j}^{(b)} f\right\|_{L^{p}\left(\mathbb{R}^{n}\right)} \leq C 2^{-\delta|j|}\|f\|_{\dot{F}_{p q}^{\alpha}}
$$

by using (3.13) in the case $j \leq 0$, and interpolating (3.12) and (3.13) in the case $j>0$, as in the case (i).

Thus by (2.24) and (3.14) we obtain

$$
\left\|\tilde{\mu}_{\Omega, \rho, \phi, \alpha, q}^{(b)} f\right\|_{L^{p}\left(\mathbb{R}^{n)}\right.} \leq \sum_{j \in \mathbb{Z}}\left\|\tilde{\mu}_{\Omega, \rho, \phi, \alpha, q, j}^{(b)} f\right\|_{L^{p}\left(\mathbb{R}^{n}\right)} \leq C W_{\Omega}\|b\|_{\Delta_{2}}\|f\|_{\dot{F}_{p q}^{\alpha}} .
$$

This completes the proof of Theorem 4(ii).

\subsection{Proof of (iii)}

We proceed to show (iii). Let $\Omega \in L \log L\left(S^{n-1}\right)$. We normalize $\Omega$ to have $\|\Omega\|_{L \log L\left(S^{n-1}\right)}=1$. Then, as in [8, pp.698-699], there is a subset $\Lambda \subset \mathbb{N} \cup\{0\}$ and a sequence of functions $\left\{\Omega_{m} ; m \in \Lambda\right.$ satisfying $0 \in \Lambda$ and the following conditions:

$$
\begin{aligned}
& \int_{S^{n-1}} \Omega_{m}\left(y^{\prime}\right) d \sigma\left(y^{\prime}\right)=0 ; \\
& \Omega\left(x^{\prime}\right)=\sum_{m \in \Lambda} \Omega_{m}\left(x^{\prime}\right) ; \\
& \left\|\Omega_{0}\right\|_{L^{2}\left(S^{n-1}\right)}+\sum_{m \in \Lambda} m\left\|\Omega_{m}\right\|_{L^{1}\left(S^{n-1}\right)} \leq C\|\Omega\|_{L \log L\left(S^{n-1}\right)} .
\end{aligned}
$$


Indeed, we just let

$$
\Lambda=\left\{m \in \mathbb{N}: \sigma\left\{2^{m-1}<|\Omega| \leq 2^{m}\right\}>2^{-4 m}\right\}
$$

and define

$$
\begin{aligned}
& \Omega_{m}(x)=\Omega(x) \chi_{\left\{2^{m-1}<|\Omega| \leq 2^{m}\right\}}(x)-\frac{1}{\sigma\left(S^{n-1}\right)} \int_{2^{m-1}<|\Omega(y)| \leq 2^{m}} \Omega(y) d \sigma(y), \\
& \Omega_{0}(x)=\Omega(x)-\sum_{m \in \Lambda \cap \mathbb{N}} \Omega_{m}(x) .
\end{aligned}
$$

For details we refer to [22].

Now for $m \in \Lambda$, by observing the proof of the case (i), we choose $\theta_{1}$ and $\theta_{2}$ very close to

$$
4 \frac{1 / \tilde{p}-1 /(2 \gamma)}{1-1 / \gamma} \cdot \frac{1 / \tilde{q}-1 /(2 \gamma)}{1-1 / \gamma}
$$

so that $\delta=\alpha / c_{1}$ for small $\alpha>0$. For large $m$, setting $\alpha=1 / m$, we obtain

$$
\left\|\mu_{\Omega_{m}, \rho, \phi, \alpha, q, f}^{(b)}\right\|_{L^{p}\left(\mathbb{R}^{n}\right)} \leq C 2^{-|j| \mid m}\left\|\Omega_{m}\right\|_{L^{1}\left(S^{n-1}\right)}\|f\|_{\dot{F}_{p q}^{1 / m}}, \quad j \in \mathbb{Z} .
$$

Next, from $\Omega_{m} \in L^{2}\left(S^{n-1}\right)$ it follows that $\Omega_{m}$ satisfies the condition in Theorem 4(ii) for any $\beta<1 / 2$. Fix $0<\beta<1 / 2$ and $\alpha_{0}>0$ with

$$
\alpha_{0}<\left(0, \min \left\{\frac{2 \beta}{c_{1} \log _{2} c_{0}} \cdot \frac{1 / \tilde{p}-1 /(2 \gamma)}{1-1 / \gamma} \cdot \frac{1 / \tilde{q}-1 /(2 \gamma)}{1-1 / \gamma}, \frac{\rho}{\log _{2} c_{0}}\right\}\right) .
$$

Let also

$$
\delta_{0}=\min \left\{\frac{\alpha_{0}}{c_{1}}, \frac{\beta \theta_{1} \theta_{2}}{2 c_{1}}+\alpha_{0} \log _{2} c_{0}\right\}
$$

in the proof of the case (ii). Then we obtain

$$
\left\|\mu_{\Omega_{m}, \rho, \phi,-\alpha_{0}, q, j}^{(b)} f\right\|_{L^{p}\left(\mathbb{R}^{n)}\right.} \leq C 2^{-\delta_{0}|j|}\left\|\Omega_{m}\right\|_{L^{2}\left(S^{n-1}\right)}\|f\|_{\dot{F}_{p q}^{-\alpha_{0}}}, \quad j \in \mathbb{Z} .
$$

Since $\frac{\alpha_{0}}{1 / m+\alpha_{0}}+\left(1-\frac{\alpha_{0}}{1 / m+\alpha_{0}}\right)=1$ and $\frac{1}{m} \cdot \frac{\alpha_{0}}{1 / m+\alpha_{0}}-\alpha_{0}\left(1-\frac{\alpha_{0}}{1 / m+\alpha_{0}}\right)=0$, an interpolation between (3.18) and (3.19) yields

$$
\begin{aligned}
& \left\|\mu_{\Omega_{m}, \rho, \phi, 0, q, j}^{(b)} f\right\|_{L^{p}\left(\mathbb{R}^{n}\right)} \\
& \quad \leq C 2^{-\left(\alpha_{0} /\left(1+m \alpha_{0}\right)+\delta_{0} /\left(1+m \alpha_{0}\right)\right)|j|}\left\|\Omega_{m}\right\|_{L^{1}\left(S^{n-1}\right)}^{\alpha_{0}\left(1 / m+\alpha_{0}\right)}\left\|\Omega_{m}\right\|_{L^{2}\left(S^{n-1}\right)}^{1 /\left(1+m \alpha_{0}\right)}\|f\|_{\dot{F}_{p q}^{0}} \\
& \quad \leq C 2^{-|j| / m} 2^{4 / \alpha_{0}}\|f\|_{\dot{F}_{p q}^{0}}, \quad j \in \mathbb{Z} .
\end{aligned}
$$

Thus, summing up the above estimate, we obtain

$$
\left\|\mu_{\Omega_{m}, \rho, \phi, 0, q}^{(b)} f\right\|_{L^{p}\left(\mathbb{R}^{n}\right)} \leq \frac{C}{1-2^{-1 / m}}\|f\|_{\dot{F}_{p q}^{0}} \leq C m\|f\|_{\dot{F}_{p q}^{0}} .
$$


Combining (3.20) with (3.16) and (3.17) and the definition of $\mu_{\Omega, 0, \rho, q}^{(b)}$, we obtain the desired estimate

$$
\left\|\mu_{\Omega, \rho, \phi, 0, q}^{(b)} f\right\|_{L^{p}\left(\mathbb{R}^{n)}\right.} \leq C\|\Omega\|_{L \log L\left(S^{n-1}\right)}\|f\|_{\dot{F}_{p q}^{0}}
$$

Thus, we are done.

\section{Proof of Theorem 3}

Here we shall relax the condition on $\alpha$ by taking advantage of a new condition on $\phi$. We use the notations in the proof of Theorem 4 , by setting $b(t) \equiv 1$ and $\gamma=\infty$. Using (1.18) and (1.19), we apply Theorem 4(i) and we obtain the conclusion of Theorem 3(i).

We go to the proof of (ii). First

$$
\begin{aligned}
\widehat{\sigma}_{t}(\xi) & =\frac{1}{t^{\rho}} \int_{B(t) \backslash B(t / 2)} \frac{\Omega\left(y^{\prime}\right)}{|y|^{n-\rho}} e^{-i \phi(|y|) y^{\prime} \cdot \xi} d y \\
& =\int_{S^{n-1}} \Omega\left(y^{\prime}\right) \frac{1}{t^{\rho}}\left(\int_{t / 2}^{t} e^{-i \phi(|y|) y^{\prime} \cdot \xi} r^{\rho-1} d r\right) d \sigma\left(y^{\prime}\right) .
\end{aligned}
$$

With a change of variables we get

$$
\begin{aligned}
B(t, \xi) & :=\frac{1}{t^{\rho}} \int_{t / 2}^{t} e^{-i \phi(|y|) y^{\prime} \cdot \xi} r^{\rho-1} d r \\
& =\frac{1}{t^{\rho}} \int_{\phi(t / 2)}^{\phi(t)} e^{-i s y^{\prime} \cdot \xi} \frac{\phi^{-1}(s)^{\rho}}{\phi^{-1}(s) \phi^{\prime}\left(\phi^{-1}(s)\right)} d s \\
& =\frac{1}{t^{\rho}} \int_{\phi(t / 2)}^{\phi(t)} e^{-i s y^{\prime} \cdot \xi} \frac{\phi^{-1}(s)^{\rho}}{s} \cdot \frac{\phi\left(\phi^{-1}(s)\right)}{\phi^{-1}(s) \phi^{\prime}\left(\phi^{-1}(s)\right)} d s \\
& =\frac{1}{t^{\rho}} \int_{\phi(t / 2)}^{\phi(t)} e^{-i s y^{\prime} \cdot \xi} \frac{\phi^{-1}(s)^{\rho}}{s} \cdot \varphi\left(\phi^{-1}(s)\right) d s .
\end{aligned}
$$

Suppose now that $t \phi^{\prime}(t)$ is increasing on $\mathbb{R}_{+}$. Then $\phi^{-1}(s) \phi^{\prime}\left(\phi^{-1}(s)\right)$ is also increasing. So by applying the second mean value theorem to the real part of the expression (4.2), we see that there exists $u$ with $\phi(t / 2)<u<\phi(t)$ such that

$$
\operatorname{Re} B(t, \xi)=\frac{1}{t^{\rho} \phi^{-1}(\phi(t / 2)) \phi^{\prime}\left(\phi^{-1}(\phi(t / 2))\right.} \int_{\phi(t / 2)}^{u} \operatorname{Re}\left(e^{-i s y^{\prime} \cdot \xi}\right) \phi^{-1}(s)^{\rho} d s
$$

Since $\phi^{-1}(s)^{\rho}$ is increasing, we have

$$
\begin{aligned}
|\operatorname{Re} B(t, \xi)| & \leq \frac{\phi^{-1}(u)^{\rho}}{t^{\rho} \phi^{-1}(\phi(t / 2)) \phi^{\prime}\left(\phi^{-1}(\phi(t / 2))\left|y^{\prime} \cdot \xi\right|\right.} \\
& \leq \frac{\phi^{-1}(\phi(t))^{\rho}}{t^{\rho}} \cdot \frac{\phi\left(\phi^{-1}(\phi(t / 2))\right)}{\phi^{-1}(\phi(t / 2)) \phi^{\prime}\left(\phi^{-1}(\phi(t / 2))\right.} \cdot \frac{1}{\phi(t / 2)\left|y^{\prime} \cdot \xi\right|} \\
& =\frac{\phi^{-1}(\phi(t))^{\rho}}{t^{\rho}} \cdot \frac{\phi(t / 2)}{t / 2 \cdot \phi^{\prime}(t / 2)} \cdot \frac{1}{\phi(t / 2)\left|y^{\prime} \cdot \xi\right|} \\
& =\frac{\phi^{-1}(\phi(t))^{\rho} \varphi(t / 2)}{t^{\rho}} \cdot \frac{1}{\phi(t / 2)\left|y^{\prime} \cdot \xi\right|} \leq C \frac{c_{0}\|\varphi\|_{\infty}}{\phi(t)\left|y^{\prime} \cdot \xi\right|} .
\end{aligned}
$$


After estimating $\operatorname{Im} B(t, \xi)$ in a similar manner, we obtain

$$
|B(t, \xi)| \leq C \frac{c_{0}\|\varphi\|_{\infty}}{\phi(t)\left|y^{\prime} \cdot \xi\right|}
$$

In the case $t \phi^{\prime}(t)$ is decreasing or $\varphi(t)$ is monotonic, we get the same estimate (4.3) in a similar way. Clearly, we have $|B(t, \xi)| \leq 1 / \rho$, and hence for any $0<\beta \leq 1,|B(t, \xi)| \leq$ $\frac{C}{\left(\phi(t)\left|y^{\prime} \cdot \xi\right|\right)^{\beta}}$. By (4.1) we get

$$
\left|\widehat{\sigma}_{t}(\xi)\right| \leq C\left(\int_{S^{n-1}} \frac{\mid \Omega\left(y^{\prime}\right)}{\left|y^{\prime} \cdot \xi^{\prime}\right|^{\beta}} d \sigma\left(y^{\prime}\right)\right) \frac{1}{(\phi(t)|\xi|)^{\beta}} .
$$

Now the rest of the proof is the same as that of the case (i).

This completes the proof of Theorem 3.

\section{Proof of Proposition 1}

The part is an appendix of the present paper, where we prove Proposition 1. Let $\psi \in \mathcal{S}\left(\mathbb{R}^{n}\right)$ (see Figure 5) be chosen so that

$$
\chi_{B(1)} \leq \psi \leq \chi_{B\left(a^{1 / 3}\right)} .
$$

Define

$$
\varphi_{k}(\xi)=\psi\left(a_{k}^{-1} \xi\right)-\psi\left(a_{k-1}^{-1} \xi\right) \quad\left(\xi \in \mathbb{R}^{n}\right) .
$$

Notice that $\operatorname{supp} \varphi_{k} \subset\left\{\xi \in \mathbb{R}^{n} ; a_{k-1} \leq|\xi| \leq a^{1 / 3} a_{k}\right\}(k \in \mathbb{Z})$ and that $\varphi_{k}(\xi)=1$ on $\left\{a^{1 / 3} a_{k-1} \leq|\xi| \leq a_{k}\right\}$. Let

$$
\Phi_{k}=\mathcal{F}^{-1} \varphi_{k}
$$

Then we see that $\left\{\Phi_{k}\right\}_{k \in \mathbb{Z}}$ is a partition of unity adapted to $\left\{a_{k}\right\}_{k \in \mathbb{Z}}$. Similarly, taking $\psi$ so that

$$
\chi_{B\left(a^{-1 / 3}\right)} \leq \psi \leq \chi_{B(1)}
$$

Figure 5 The graph of $\psi_{j}$.

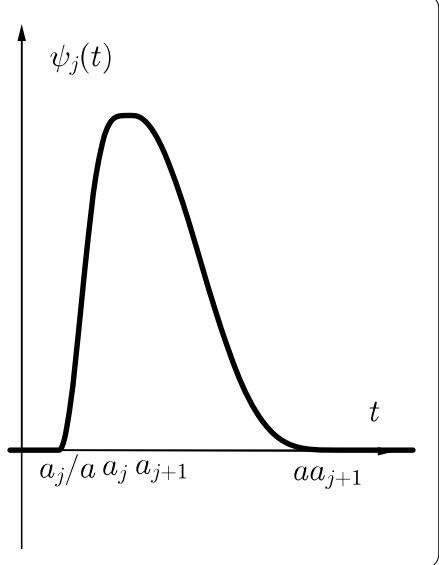


and setting

$$
\varphi_{k}(\xi)=\psi\left(a_{k+1}^{-1} \xi\right)-\psi\left(a_{k}^{-1} \xi\right) \quad\left(\xi \in \mathbb{R}^{n}\right)
$$

we obtain another partition of unity $\left\{\Psi_{k}\right\}_{k \in \mathbb{Z}}$ adapted to $\left\{a_{k}\right\}_{k \in \mathbb{Z}}$ satisfying supp $\widehat{\Psi}_{k} \subset\{\xi \in$ $\left.\mathbb{R}^{n} ; a_{k} / a^{1 / 3} \leq|\xi| \leq a_{k+1}\right\}(k \in \mathbb{Z})$ and $\widehat{\Psi}_{k}(\xi)=1$ on $\left\{a_{k} \leq|\xi| \leq a_{k+1} / a^{1 / 3}\right\}$. Note that $\left\{a_{k} \leq\right.$ $\left.|\xi| \leq a^{2 / 3} a_{k}\right\} \subset\left\{a_{k} \leq|\xi| \leq a_{k+1} / a^{1 / 3}\right\}$. Let us take a function $\Theta \in \mathcal{S}$ so that $\operatorname{supp}(\mathcal{F} \Theta) \subset$ $B\left(a^{1 / 3} / 2-1 / 2\right)$. Consider

$$
f_{k}(x)=f_{k}\left(x_{1}, x_{2}, \ldots, x_{n}\right)=\exp \left(i \frac{\left(a^{1 / 3}+a^{2 / 3}\right)}{2} a_{k} x_{1}\right) \Theta\left(a_{k} x\right) \quad\left(x \in \mathbb{R}^{n}\right) .
$$

Then we have

$$
\mathcal{F} f_{k}(\xi)=a_{k}^{-n} \mathcal{F} \Theta\left(\frac{\xi}{a_{k}}-\frac{\left(a^{1 / 3}+a^{2 / 3}\right)}{2} \mathbf{e}_{1}\right) \quad\left(\xi \in \mathbb{R}^{n}\right),
$$

where $\mathbf{e}_{1}=(1,0, \ldots, 0)$. It follows that supp $\mathcal{F} f_{k} \subset\left\{a^{1 / 3} a_{k} \leq|\xi| \leq a^{2 / 3} a_{k}\right\}$. Hence we have

$$
\Phi_{j} * f_{k}(x)=\delta_{(j-1) k} f_{k}(x) \quad \text { and } \quad \Psi_{j} * f_{k}(x)=\delta_{j k} f_{k}(x)
$$

where

$$
\delta_{j k}= \begin{cases}1 & (j=k), \\ 0 & (j \neq k)\end{cases}
$$

for $j, k \in \mathbb{Z}$.

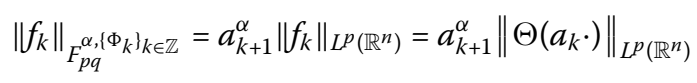

and

$$
\left\|f_{k}\right\|_{F_{p q+}^{\alpha,}\left\{\Psi_{k}\right\}_{k \in \mathbb{Z}}}=a_{k}^{\alpha}\left\|f_{k}\right\|_{L^{p}\left(\mathbb{R}^{n)}\right.}=a_{k}^{\alpha}\left\|\Theta\left(a_{k} \cdot\right)\right\|_{L^{p}\left(\mathbb{R}^{n}\right)} .
$$

Since the two norms are assumed equivalent, we obtain

$$
\frac{a_{k+1}}{a_{k}} \leq C_{0}
$$

for some $C_{0}>1$. Since $\frac{a_{k+1}}{a_{k}} \geq a$, we have $C_{0}>a$.

Thus we have proved the first part of our proposition. We proceed to the second part. Let $\left\{a_{k}\right\}_{k \in \mathbb{Z}}$ be a lacunary sequence of positive numbers with $1<a \leq a_{k+1} / a_{k} \leq C_{0}(k \in \mathbb{Z})$, and let $\left\{\Phi_{k}\right\}_{k \in \mathbb{Z}}$ be a partition of unity adapted to $\left\{a_{k}\right\}_{k \in \mathbb{Z}}$.

Now we can define the classical homogeneous Triebel-Lizorkin spaces as follows: Let $\psi \in \mathcal{S}\left(\mathbb{R}^{n}\right)$ be chosen so that $\chi_{B\left(a^{2 / 3}\right)} \leq \psi \leq \chi_{B(a)}$. Define

$$
\varphi_{k}(\xi)=\psi\left(a^{-k} \xi\right)-\psi\left(a^{-k+1} \xi\right) \quad\left(\xi \in \mathbb{R}^{n}\right)
$$

Notice that $\varphi_{k}(\xi)=1$ on $\left\{a^{k} \leq|\xi| \leq a^{k+\frac{2}{3}}\right\}$. 
Define

$$
\|f\|_{\dot{F}_{p q}^{\alpha}}=\left\|\left(\sum_{j=-\infty}^{\infty}\left|a^{\alpha j} \mathcal{F}^{-1} \varphi_{j} * f\right|^{q}\right)^{1 / q}\right\|_{L^{p}}
$$

Let us prove

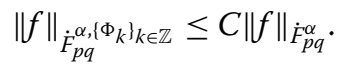

For each $k \in \mathbb{Z}$, we choose $m_{k} \in \mathbb{Z}$ so that

$$
a^{m_{k}} \leq a_{k}<a^{m_{k}+1}
$$

Combining with $a^{m_{k}+1} \leq a a_{k} \leq a_{k+1}$, we get $m_{k+1} \geq m_{k}+1$. And combining with $a_{k+1} / a_{k} \leq$ $C_{0}$, we have $m_{k+1}-m_{k} \leq 1+\log _{a} C_{0}$. Furthermore we have

$$
\Phi_{k}=\Phi_{k} * \sum_{l=m_{k-1}}^{m_{k+1}+1} \varphi_{l}
$$

Consequently, we obtain

$$
\begin{aligned}
& \|f\|_{\dot{F}_{p q}^{\alpha,\left\{\Phi_{k}\right\}_{k \in \mathbb{Z}}}}=\left\|\left(\sum_{k \in \mathbb{Z}} a_{k}^{\alpha q}\left|\Phi_{k} * f\right|^{q}\right)^{1 / q}\right\|_{L^{p}\left(\mathbb{R}^{n}\right)} \\
& =\left\|\left(\sum_{k \in \mathbb{Z}} a_{k}^{\alpha q}\left|\Phi_{k} * \sum_{l=m_{k-1}}^{m_{k+1}+1} \varphi_{l} * f\right|^{q}\right)^{1 / q}\right\|_{L^{p}\left(\mathbb{R}^{n}\right)} \\
& \leq\left\|\left(\sum_{k \in \mathbb{Z}} a_{k}^{\alpha q}\left|\Phi_{k} * \sum_{l=m_{k-1}}^{m_{k+1}+1} \varphi_{l} * f\right|^{q}\right)^{1 / q}\right\|_{L^{p}\left(\mathbb{R}^{n}\right)} .
\end{aligned}
$$

We now invoke the Plancherel-Polya-Nikolskij inequality: We have

$$
\left|\sum_{l=m_{k-1}}^{m_{k+1}+1} \varphi_{l} * f(x)\right| \leq C\left(1+\left|a^{m_{k+1}+1}(x-y)\right|\right)^{n} M\left[\sum_{l=m_{k-1}}^{m_{k+1}+1} \varphi_{l} * f\right](y) .
$$

Using Plancherel's theorem, the assumption $\left|\xi^{\beta} \partial^{\beta} \widehat{\Phi}_{k}(\xi)\right| \leq C_{\beta}$ for all $\beta$ and that supp $\widehat{\Phi}_{k} \subset$ $\left\{a_{k-1} \leq|\xi| \leq a_{k+1}\right\}$, we get

$$
\begin{aligned}
& \int_{\mathbb{R}^{n}}\left(1+\left|a^{m_{k+1}+1} x\right|\right)^{n}\left|\Phi_{k}(x)\right| d x \\
& \quad \leq C \int_{\mathbb{R}^{n}}\left(1+\left|a_{k+1} x\right|\right)^{n}\left|\Phi_{k}(x)\right| d x \\
& \quad \leq C\left(\int_{\mathbb{R}^{n}}\left(1+\left|a_{k+1} x\right|\right)^{4 n}\left|\Phi_{k}(x)\right|^{2} d x\right)^{1 / 2}\left(\int_{\mathbb{R}^{n}}\left(1+\left|a_{k+1} x\right|\right)^{-2 n} d x\right)^{1 / 2} \\
& \quad \leq C a_{k}^{-n / 2}\left(\int_{\mathbb{R}^{n}}\left(\left|\widehat{\Phi}_{k}(\xi)\right|^{2}+a_{k+1}^{4 n}\left|\nabla^{n} \widehat{\Phi}_{k}(\xi)\right|^{2}\right) d \xi\right)^{1 / 2}
\end{aligned}
$$




$$
\begin{aligned}
& \leq C a_{k}{ }^{-n / 2}\left(\int_{a_{k-1} \leq|\xi| \leq a_{k+1}}\left(\left|\widehat{\Phi}_{k}(\xi)\right|^{2}+a_{k+1}^{4 n}|\xi|^{-4 n}\right) d \xi\right)^{1 / 2} \\
& \leq C .
\end{aligned}
$$

Hence, it follows that

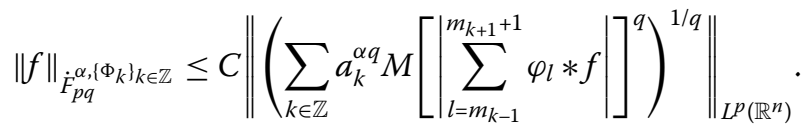

By the Fefferman-Stein vector-valued maximal inequality (see [23]), we obtain

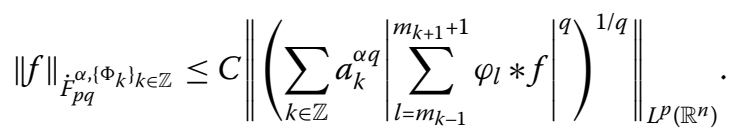

If we use $\left(a_{1}+a_{2}+\cdots+a_{N}\right)^{q} \leq N^{q}\left(a_{1}^{q}+a_{2}^{q}+\cdots+a_{N}^{q}\right)$, then we obtain

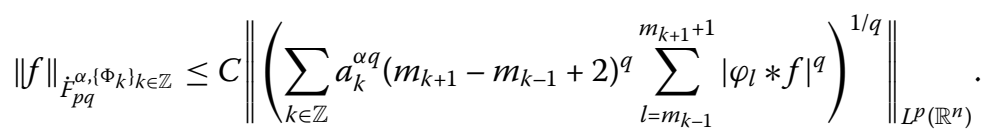

Noting $m_{k+1}-m_{k} \geq 1, m_{k+1}-m_{k} \leq 1+\log _{a} C_{0}$ and that $a^{m_{k-1}+1} \leq a^{m_{k}} \leq a_{k}<a^{m_{k}+1} \leq$ $a^{m_{k-1}+1+\left[\log _{a} C_{0}\right]}$, we conclude

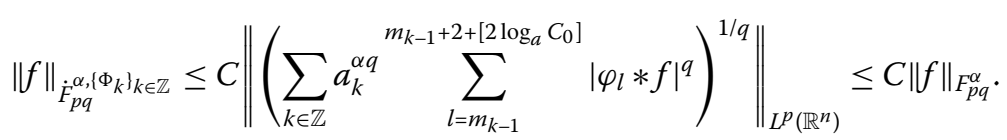

Let us prove the reverse inequality. For each $k \in \mathbb{Z}$, we can choose $\ell_{k} \in \mathbb{Z}$ so that

$$
a_{\ell_{k}} \leq a^{k-1} \leq a^{k+1} \leq a_{\ell_{k}+3}
$$

Then we have

$$
\varphi_{k}=\varphi_{k}\left(\Phi_{\ell_{k}}+\Phi_{\ell_{k}+1}+\Phi_{\ell_{k}+2}+\Phi_{\ell_{k}+3}\right)
$$

Notice that

$$
\sup _{l \in \mathbb{Z}} \sharp\left\{k: \ell_{k}=l\right\} \leq 3 \log _{a} C_{0}
$$

because $a_{l+3} / a_{l} \leq C_{0}^{3}$. Thus, it follows that

$$
\begin{aligned}
\|f\|_{\dot{F}_{p q}^{\alpha}} & =\left\|\left(\sum_{k=-\infty}^{\infty}\left|a^{\alpha k} \mathcal{F}^{-1} \varphi_{k} * f\right|^{q}\right)^{1 / q}\right\|_{L^{p}} \\
& =\left\|\left(\sum_{k=-\infty}^{\infty}\left|a^{\alpha k} \mathcal{F}^{-1} \varphi_{k} *\left(\mathcal{F}^{-1} \Phi_{\ell_{k}}+\cdots+\mathcal{F}^{-1} \Phi_{\ell_{k}+3}\right) * f\right|^{q}\right)^{1 / q}\right\|_{L^{p}} \\
& \leq C\left\|\left(\sum_{k=-\infty}^{\infty} M\left[\left|a^{\alpha k}\left(\mathcal{F}^{-1} \Phi_{\ell_{k}}+\cdots+\mathcal{F}^{-1} \Phi_{\ell_{k}+3}\right) * f\right|\right]^{q}\right)^{1 / q}\right\|_{L^{p}} .
\end{aligned}
$$


Again by the Fefferman-Stein vector-valued maximal inequality (see [23]), we obtain

$$
\begin{aligned}
& \|f\|_{\dot{F}_{p q}^{\alpha}} \leq C\left\|\left(\sum_{k=-\infty}^{\infty}\left|a^{\alpha k}\left(\mathcal{F}^{-1} \Phi_{\ell_{k}}+\cdots+\mathcal{F}^{-1} \Phi_{\ell_{k}+3}\right) * f\right|^{q}\right)^{1 / q}\right\|_{L^{p}} \\
& \leq C\left\|\left(\sum_{k=-\infty}^{\infty}\left(\left|a_{\ell_{k}}^{\alpha} \mathcal{F}^{-1} \Phi_{\ell_{k}} * f\right|+\cdots+\left|a_{\ell_{k}+3}^{\alpha} \mathcal{F}^{-1} \Phi_{\ell_{k}+3} * f\right|\right)^{q}\right)^{1 / q}\right\|_{L^{p}} \\
& \leq C\|f\|_{\dot{F}_{p q}^{\alpha,\left\{\Phi_{k}\right\}_{k} \in \mathbb{Z}}} .
\end{aligned}
$$

This completes the proof of our proposition.

\section{Competing interests}

The authors declare that they have no competing interests.

\section{Authors' contributions}

All authors contributed equally to the writing of this paper. All authors read and approved the final manuscript.

\section{Author details}

'Department of Mathematics and Information Science, Tokyo Metropolitan University, Minami-Ohsawa 1-1, Hachioji, 192-0397, Japan. ${ }^{2}$ Research Center for Mathematical Sciences, Kwansei Gakuin University, Gakuen 2-1, Sanda, 669-1337, Japan.

\section{Acknowledgements}

This work was partially supported by Grant-in-Aid for Scientific Research (C) No. 23540228, Japan Society for the Promotion of Science and Grant-in-Aid for Young Scientists (B) No. 24740085, Japan Society for the Promotion of Science.

\section{Received: 1 March 2014 Accepted: 6 May 2014 Published: 04 Jun 2014}

\section{References}

1. Stein, EM: On the function of Littlewood-Paley, Lusin and Marcinkiewicz. Trans. Am. Math. Soc. 88, 430-466 (1958)

2. Hörmander, L: Estimates for translation invariant operators in $L^{P}$ spaces. Acta Math. 104, 93-140 (1960)

3. Lu, SZ: Marcinkiewicz integrals with rough kernels. Front. Math. China 3, 1-14 (2008)

4. Triebel, H: Theory of Function Spaces. Birkhäuser, Basel (1983)

5. Grafakos, L: Modern Fourier Analysis, 2nd edn. Springer, Berlin (2009)

6. Chen, J, Fan, D, Ying, Y: Singular integral operators on function spaces. J. Math. Anal. Appl. 276, 691-708 (2002)

7. Si, ZY, Wang, LN, Jiang, YS: Fractional type Marcinkiewicz integral on Hardy spaces. J. Math. Res. Expo. 31, 233-241 (2011)

8. Al-Salman, A, Al-Qassem, H, Cheng, L, Pan, Y: $L^{p}$ bounds for the function of Marcinkiewicz. Math. Res. Lett. 9, 697-700 (2002)

9. Xue, Q, Yabuta, K, Yan, J: Fractional type Marcinkiewicz integral operators on function spaces. Forum Math. (2014) doi:10.1515/forum-2013-0200

10. Al-Qassem, HM, Pan, Y: On rough maximal operators and Marcinkiewicz integrals along submanifolds. Stud. Math. 190(1), 73-98 (2009)

11. Duoandikoetxea, J, Rubio de Francia, JL: Maximal and singular integral operators via Fourier transform estimates. Invent. Math. 84, 541-561 (1986)

12. Fan, D, Pan, Y: A singular integral operators with rough kernel. Proc. Am. Math. Soc. 125, 3695-3703 (1997)

13. Fan, D, Pan, Y: Singular integral operators with rough kernels supported by subvarieties. Am. J. Math. 119, 799-839 (1997)

14. Fefferman, R: A note on singular integrals. Proc. Am. Math. Soc. 74, 266-270 (1979)

15. Burenkov, VI, Guliyev, VS, Serbetci, A, Tararykova, TV: Necessary and sufficient conditions for the boundedness of genuine singular integral operators in local Morrey-type spaces. Eurasian Math. J. 1(1), 32-53 (2010)

16. Komori, Y, Matsuoka, K, Nakai, E, Sawano, Y: Integral operators on $B^{\sigma}$ spaces. Rev. Mat. Complut. 26(1), 1-32 (2013)

17. Matsuoka, K, Nakai, E: Fractional integral operators on $B^{p, \lambda}$ with Morrey-Campanato norms. In: Function Spaces IX. Banach Center Publ., vol. 92, pp. 249-264. Polish Acad. Sci. Inst. Math, Warsaw (2011)

18. Al-Qassem, HM: Weighted $L^{p}$ boundedness for the function of Marcinkiewicz. Kyungpook Math. J. 46, 31-48 (2006)

19. Ding, $Y, X u e, Q$, Yabuta, $K$ : A remark to the $L^{2}$ boundedness of parametric Marcinkiewicz integral. J. Math. Anal. Appl. 378, 691-697 (2012)

20. Ding, Y, Fan, D, Pan, Y: On Littlewood-Paley functions and singular integrals. Hokkaido Math. J. 29, 537-552 (2000)

21. Ding, Y, Xue, Q, Yabuta, K: Boundedness of the Marcinkiewicz integrals with rough kernel associated to surfaces. Tohoku Math. J. 62, 233-262 (2010)

22. Al-Salman, A: On the $L^{2}$ boundedness of parametric Marcinkiewicz integral operator. J. Math. Anal. Appl. 375, 745-752 (2011)

23. Fefferman, C, Stein, EM: Some maximal inequalities. Am. J. Math. 93, 107-115 (1971) 
10.1186/1029-242X-2014-232

Cite this article as: Sawano and Yabuta: Fractional type Marcinkiewicz integral operators associated to surfaces. Journal of Inequalities and Applications 2014, 2014:232

Submit your manuscript to a SpringerOpen ${ }^{\circ}$ journal and benefit from:

- Convenient online submission

- Rigorous peer review

- Immediate publication on acceptance

Open access: articles freely available online

- High visibility within the field

- Retaining the copyright to your article

Submit your next manuscript at $\gg$ springeropen.com 